\title{
Sbottoms as probes to MSSM with nonholomorphic soft interactions
}

\author{
Utpal Chattopadhyay, ${ }^{a}$ AseshKrishna Datta, ${ }^{b}$ Samadrita Mukherjee ${ }^{a}$ and \\ Abhaya Kumar Swain ${ }^{a}$ \\ ${ }^{a}$ School of Physical Sciences, Indian Association for the Cultivation of Science, \\ $2 A \&$ B Raja S.C. Mullick Road, Jadavpur, Kolkata 700 032, India \\ ${ }^{b}$ Harish-Chandra Research Institute, HBNI, \\ Chhatnag Road, Jhusi, Allahabad-211019, India \\ E-mail: tpuc@iacs.res.in, asesh@hri.res.in, tpsm9@iacs.res.in, \\ tpaks@iacs.res.in
}

ABSTRACT: Presence of nonholomorphic soft SUSY breaking terms is known to be a possibility in the popular setup of the Minimal Supersymmetric Standard Model (MSSM). It has been shown that such a scenario known as NonHolomorphic Supersymmetric Standard Model (NHSSM) could remain 'natural' (i.e., not fine-tuned) even in the presence of a rather heavy higgsino-like LSP. However, it turns out that distinguishing such a scenario from the MSSM is unlikely to be an easy task, in particular at the Large Hadron Collider (LHC). In a first study of such a scenario at colliders (LHC), we explore a possible way that focuses on the sbottom phenomenology. This exploits the usual $\tan \beta$-dependence (enhancement) of the bottom Yukawa coupling but reinforced/altered in the presence of non-vanishing nonholomorphic soft trilinear parameter $A_{b}^{\prime}$. For a given set of masses of the sbottom(s) and the light electroweakinos (LSP, lighter chargino etc.) which are known from experiments, the difference between the two scenarios could manifest itself via event rate in the $2 b$-jets $+\mathbb{E}_{T}$ final state, which could be characteristically different from its MSSM expectation. Impact on the phenomenology of the stops at the LHC is also touched upon.

KEywords: Supersymmetry Phenomenology

ArXiv ePrint: 1809.05438

Open AcCess, (c) The Authors.

Article funded by $\mathrm{SCOAP}^{3}$.

https://doi.org/10.1007/JHEP10(2018)202 


\section{Contents}

1 Introduction 1

2 Nonholomorphic soft terms and their phenomenological implications 4

3 Sbottom phenomenology in the NHSSM (vis-a-vis the MSSM)

3.1 Interactions with electroweakinos 8

$\begin{array}{lll}3.2 & \text { Masses, mixings and decays of the lighter sbottom } & 12\end{array}$

$\begin{array}{lll}3.3 & \text { Pair-productions of sbottoms at the LHC } & 17\end{array}$

$\begin{array}{lll}3.3 .1 & \text { The case with the lighter sbottom } & 18\end{array}$

3.3.2 Impact of including the heavier sbottom 22

3.4 Implication for stop searches 26

$\begin{array}{llr}4 & \text { Conclusions } & 28\end{array}$

\section{Introduction}

The discovery of Higgs boson at the Large Hadron Collider (LHC) of CERN with mass $125.09 \pm 0.21$ (stat.) \pm 0.11 (syst.) GeV [1, 2] established the Standard Model (SM) of particle physics as one of the most successful scientific pursuits of mankind. There are, however, a host of theoretical issues along with some experimental facts that cannot be addressed while staying within the SM. The gauge hierarchy problem, baryogenesis, the existence of mass for neutrinos, the absence of a particle dark matter (DM) candidate are a few of the important issues that motivate us to explore scenarios of physics Beyond the SM (BSM). Supersymmetry (SUSY) [3-10] remains to be the most attractive framework for pursuing BSM physics.

The fact that the Higgs boson has been found to have a mass well within the upper limit $(\sim 135 \mathrm{GeV})$ predicted by the Minimal Supersymmetric Standard Model (MSSM) $[6,7]$ is something hard to ignore. On the one hand, this has pushed the lower bound on the masses of the hypothesized SUSY excitations to higher values, a fact that is especially true for models employing simpler mechanisms to break SUSY. Such scenarios, thus, require a greater degree of fine-tuning and hence are less 'natural' [11-14]. On the other hand, even after the $13 \mathrm{TeV}$ run of the LHC, a definitive signature of SUSY is yet to be established $[15,16]$. The null observations have for sure put serious constraints on various SUSY models. These together prompt one to explore generic SUSY models with low finetuning [17-31] and looking for their signals in unexplored and perhaps, not so conventional channels. Such models thus may still survive the latest experimental constraints, but could become sensitive to ongoing and future LHC searches. Additionally, models that may have interesting implications for the precision observables, flavor physics related signatures or those that could offer a suitable DM candidate would undoubtedly be worth pursuing. 
In the MSSM, SUSY breaking is realized by soft SUSY breaking interactions where, apart from the mass terms, all the other terms are holomorphic in nature. However, there is hardly any restriction to include appropriate nonholomorphic (NH) SUSY breaking interactions [30, 32-37]. It was shown in several works [32, 34, 38, 39] that in the absence of any gauge singlet fields, certain nonholomorphic SUSY breaking terms may become characteristically soft so that the MSSM may additionally include soft terms like $A_{f}^{\prime} \phi^{2} \phi^{*}$ and $\mu^{\prime} \psi \psi{ }^{1}$ The resulting scenario is broadly known as the NonHolomorphic Supersymmetric Standard Model (NHSSM).

Implications of the NH soft terms had been analyzed in scenarios with universal boundary conditions at a high scale [40-44]. These used renormalization group (RG) evolutions of various input parameters to the weak scale for studying the SUSY spectra, impact on the Higgs boson mass, involved fine-tuning, various phenomenological observables in rare $B$-decays (viz., $\operatorname{BR}\left(B \rightarrow X_{s}+\gamma\right), \operatorname{BR}\left(B_{s} \rightarrow \mu^{+} \mu^{-}\right)$), issues pertaining to the dark matter, $\mathrm{CP}$-violating effects etc. Analyses that were inspired by the so-called phenomenological MSSM (pMSSM)-like setup [45] (where the input values of the soft SUSY breaking parameters are provided at the electroweak scale instead) are presented in [46, 47]. The first of these two works [46] demonstrated the enhancement of muon $g-2$ in models that involve NH terms. The latter one [47] made a detailed study on the Charge and Color Breaking (CCB) vacua while including appropriate $\mathrm{NH}$ soft breaking terms. It is noted that in a formulation of hidden sector $F$-type SUSY breaking, such NH terms may be suppressed by the scale of mediation [32]. Thus, supergravity [48-52] scenarios have NH soft terms that are suppressed by the Planck mass. Recently, authors of reference [33] analyzed an NH scenario in a minimal Gauge Mediated SUSY Breaking (mGMSB) framework [6, 53] where the scale of mediation is reasonably small. However, like all the analyses to date with $\mathrm{NH}$ terms, we will remain agnostic about the source/mechanism of such suppressions and consider the parameters (at the electroweak scale) associated with $\mathrm{NH}$ terms to have similar strengths as that of the holomorphic soft parameters. ${ }^{2}$

It is now known [46] that the NH soft trilinear parameters $\left(A_{f}^{\prime}\right)$ may significantly alter the left-right (L-R) mixing of squarks and sleptons while the NH soft higgsino mass term $\left(\mu^{\prime}\right)$ may induce crucial changes in the compositions of the electroweakinos. In particular, as we shall see in section 2, the masses of the higgsino-like neutralinos would go as $\mu+\mu^{\prime}$ at the tree level. The fact that the Higgs potential in the NHSSM receives a contribution from the superpotential parameter ' $\mu$ ' (as in the MSSM), but not from $\mu^{\prime}$, gives rise to the possibility that a scenario with relatively heavy higgsino-like neutralinos could still have a small enough ' $\mu$ ' [46] and hence a low degree of tree-level electroweak fine-tuning $[23,25,28,31,57-63]$. Such a heavy higgsino $(\sim 1 \mathrm{TeV})$ could be the Lightest Supersymmetric Particle (LSP) and a viable DM candidate that could satisfy the observed relic density from the Planck data [64].

As it may be expected, in the presence of soft $\mathrm{NH}$ terms, the mass-spectra and the strength of interaction vertices involving SUSY excitations could undergo significant

\footnotetext{
${ }^{1}$ There can be other possible NH terms also like $\lambda \psi$ [32]. However, such a term with chiral fermion and gaugino mass mixing is not allowed in the MSSM particle content.

${ }^{2}$ Non-standard SUSY breaking was also studied with $R$-parity violating NH soft SUSY breaking terms in the MSSM framework in references [54-56].
} 
changes via the altered mass (and hence the diagonalizing) matrices of the sfermions and electroweakinos. On the other hand, gauge and/or Yukawa couplings may have dominant roles in determining the strength of these vertices depending on the composition of sparticles. For example, as we will see in section 3.1 , the strength of the vertices $\tilde{b}_{i}-b-\tilde{\chi}_{j}^{0}$ and $\tilde{t}_{i}-t-\tilde{\chi}_{j}^{0}$ are mostly powered by $y_{b}$ and $y_{t}$, respectively when the involved neutralino is higgsino-like, while the same are governed by gauge couplings when the neutralino is a gaugino-dominated one. Furthermore, the coupling strength of a sfermion to a chargino depends crucially on the chiral admixture the sfermion possesses. One also finds that, among the NH soft trilinear parameters $A_{f}^{\prime}$ (see section 2), the lepton (tau)- and the down (bottom)-type ones $\left(A_{\tau}^{\prime}\right.$ and $\left.A_{b}^{\prime}\right)$ might contribute dominantly to the L-R mixings of scalars [46] for an enhanced $\tan \beta$ where $\tan \beta$ is the ratio of vevs $\left(\frac{v_{2}}{v_{1}}\right)$ of the two Higgs doublets. As a result, the NH soft parameters $A_{\tau, b}^{\prime}$ could induce more L-R mixing in the stau and the sbottom sectors, respectively than what is contributed by $A_{t}^{\prime}$ of similar strength toward mixing in the stop sector. In this work we are interested in the phenomenology of the squarks from the third generation, in particular, the sbottom sector and hence would consider only $A_{b}^{\prime}$ which is non-vanishing.

Notably enough, there is another source in the NHSSM that could alter the strength of the interaction vertices significantly. The bottom Yukawa coupling $y_{b}$ could receive large radiative correction in the presence of non-vanishing $A_{b}^{\prime}$ when $\tan \beta$ is large. This is over and above the usual $\tan \beta$-enhancement that $y_{b}$ enjoys due to radiative corrections in the MSSM [65-70]. Such an effect arises in the NHSSM essentially from the coupling of the down-type sfermions (sbottoms) to the up-type Higgs boson triggered by soft trilinear $\mathrm{NH}$ interaction terms (viz., $A_{b}^{\prime}$ ). In contrast, consequences of $A_{t}^{\prime}$ in the stop mass-squared matrix is $\tan \beta$-suppressed and is practically subdominant in the background of a large holomorphic trilinear coupling $A_{t}$ that is required to obtain the SM-like Higgs boson mass in the right range. Also, the impact of $A_{t}^{\prime}$ on $y_{t}$ via radiative corrections is small. Given that the NHSSM setup cannot alter the SM-like Higgs boson mass as obtained in the MSSM any significantly, the requirement of a relatively large $\tan \beta$ would still be intact in the NHSSM. This then implies that the NHSSM-specific effects would, in general, be more pronounced in the phenomenology of the sbottoms when compared to that of the stops. There is, however, one subtle exception to this when (an altered) $y_{b}$ could take part in the decay of the stop squarks.

In this backdrop, already intensified searches at the LHC for the squarks from the third generation find added relevance and, from the viewpoint of the NHSSM, sbottoms take the front seat. Given that the masses and the compositions of the sbottoms predominantly control its (pair) production cross sections and decays, a detailed analysis of these by looking into their mutual relationships in the NHSSM framework would be an important first step in deciphering it at an actual experiment. We thus undertake an exploratory study of the pair-production of sbottoms at the ongoing $13 \mathrm{TeV}$ run of the LHC. We prefer a scenario with relatively small values of higgsino mass parameter ' $\mu$ ' (appearing in the superpotential) to ensure a 'natural' setup. If the soft NH parameter $\mu^{\prime}$ is not too large, this could ensure the lighter neutralinos and the lighter chargino to be higgsino-like thus bringing into the picture a much altered $y_{b}$ (characteristic of the NHSSM) in their interactions 
with the sbottoms. As a result, rates (cross section times the effective branching fraction or 'yields') in various final states would start to differ from their MSSM expectations. In the present study, we adopt the final state with $2 b$-jets + missing transverse energy $\left(\mathbb{E}_{T}\right)$ as the reference one.

The present work is organized as follows. In section 2 we discuss the salient aspects of the NHSSM scenario with reference to the nonholomorphic soft terms, their key impacts on the sfermion and the electroweakino sectors and on the running of the bottom quark masses (Yukawa couplings). Section 3 is devoted to a comparative discussion on the nature of the sbottoms, their decays and the resulting yields in our chosen final state as found in the NHSSM and the MSSM. A brief discussion on the limited but important impact of the NHSSM scenario on the search for stops at the LHC is also included. In section 4 we conclude.

\section{Nonholomorphic soft terms and their phenomenological implications}

As discussed in reference [46], one may consider specific nonholomorphic interactions which, in the absence of a gauge singlet field, as in the case of MSSM, may be regarded as soft SUSY breaking terms. The nonholomorphic cubic scalar interactions and bilinear fermionic mass terms also potentially fall into the class of soft SUSY breaking ones. Considering $X$ and $\Phi$ to be chiral superfields, the NH soft $D$-term contributions go like $\frac{1}{M^{3}}\left[X X^{*} \phi^{2} \phi^{*}\right]_{D}$ and $\frac{1}{M^{3}}\left[X X^{*} D^{\alpha} \phi D_{\alpha} \phi\right]_{D}$ and lead to nonholomorphic terms (e.g., $\phi^{2} \phi^{*}$ and $\psi \psi$ ) in the Lagrangian [32]. The coefficients of both $\phi^{2} \phi^{*}$ and $\psi \psi$ terms are proportional to $\frac{|F|^{2}}{M^{3}}$, where $|F|$ is the vev of the auxiliary field components of $X$. In a scenario with hidden sector SUSY breaking, nonholomorphic trilinear terms and higgsino mass term go as $\sim \frac{m_{W}^{2}}{M}$ where $M$ is the scale of mediation of SUSY breaking and can be as large as the Planck scale $\left(M_{P}\right)$. Thus, the NH soft terms are highly suppressed in supergravity type scenarios. In contrast, such terms can become phenomenologically relevant [33] in a scenario like the mGMSB with a characteristically low scale of mediation of SUSY breaking. As mentioned in the Introduction, considering the fact that the NH interactions could cause a significant change in phenomenology, we consider such terms in a model-independent way so that they can have strengths similar to that of the holomorphic soft terms.

The parts of the Lagrangian containing the $\mathrm{NH}$ terms are given by

$$
\begin{aligned}
-\mathcal{L}_{\text {soft }}^{\prime \phi^{2} \phi^{*}} & =\tilde{q} \cdot h_{d}^{*} \mathbf{A}_{\mathbf{u}}^{\prime} \tilde{u}^{*}+\tilde{q} \cdot h_{u}^{*} \mathbf{A}_{\mathbf{d}}^{\prime} \tilde{d}^{*}+\tilde{\ell} \cdot h_{u}^{*} \mathbf{A}_{\ell}^{\prime} \tilde{e}^{*}+\text { h.c. }, \\
-\mathcal{L}_{\text {soft }}^{\prime \psi \psi} & =\mu^{\prime} \tilde{h}_{u} \cdot \tilde{h}_{d},
\end{aligned}
$$

where $A_{u, d, \ell}^{\prime}$ are the NH soft trilinear parameters corresponding to the up, down and lepton sectors, respectively and $\mu^{\prime}$ is the $\mathrm{NH}$ soft higgsino mass parameter. We note that the association of the up and down types of Higgs fields are reversed when compared to the holomorphic trilinear terms so as to have the correct hypercharge assignments.

Presence of the NH soft trilinear terms containing $A_{f}^{\prime}$ modifies the off-diagonal terms of the tree-level sfermion mass-squared matrices of the MSSM. Thus, in the NHSSM, the 
generic tree-level mass-squared matrix for the sfermions is given by

$$
M_{\tilde{f}}^{2}=\left(\begin{array}{cc}
m_{\tilde{f_{L}}}^{2}+\left(\frac{1}{2}-\frac{2}{3} \sin ^{2} \theta_{W}\right) m_{Z}^{2} \cos 2 \beta+m_{f}^{2} & -m_{f}\left(A_{f}-\left(\mu+A_{f}^{\prime}\right) R_{\beta}\right) \\
-m_{f}\left(A_{f}-\left(\mu+A_{f}^{\prime}\right) R_{\beta}\right) & m_{\tilde{f_{R}}}^{2}+\frac{2}{3} \sin ^{2} \theta_{W} m_{Z}^{2} \cos 2 \beta+m_{f}^{2}
\end{array}\right),
$$

where $m_{\tilde{f}_{L, R}}$ are the soft SUSY breaking masses for the left- and the right-chiral sfermions, $m_{f}$ is the mass of the corresponding fermion, $A_{f}$ are the corresponding soft trilinear parameters while ' $\mu$ ' is the holomorphic higgsino mass parameter appearing in the superpotential. $R_{\beta}=\cot \beta(\tan \beta)$ for the up-type squark (down-type squark, slepton) masssquared matrices.

As can be seen, when compared to the MSSM case, ' $\mu$ ' in the off-diagonal term is now replaced by $\mu+A_{f}^{\prime}$. At this point, we must take due note of the fact that, unlike in the MSSM, a contribution from the trilinear $(\mathrm{NH})$ soft term $A_{f}^{\prime}$ appears in a product with $R_{\beta}$ and hence could get enhanced by $\tan \beta$ for the tau and the bottom sectors. It may also be noted that, in the viable range of $A_{t}^{\prime}$, it is possible to obtain some $\mathrm{NH}$ contribution to the radiative corrections to the mass of the SM-like Higgs boson in spite of a $\tan \beta$ suppression [46].

The electroweakino sector is the other entity that gets affected at the tree level in the presence of NH terms. When compared to the MSSM case, the entries in the higgsino block of the neutralino and the chargino mass matrices undergo the modification $\mu \rightarrow \mu+\mu^{\prime}[46]$ as given by

$$
\begin{gathered}
M_{\chi^{0}}=\left(\begin{array}{cccc}
M_{1} & 0 & -m_{Z} \cos \beta \sin \theta_{W} & m_{Z} \sin \beta \sin \theta_{W} \\
0 & M_{2} & m_{Z} \cos \beta \cos \theta_{W} & -m_{Z} \sin \beta \cos \theta_{W} \\
-m_{Z} \cos \beta \sin \theta_{W} & m_{Z} \cos \beta \cos \theta_{W} & 0 & -\left(\mu+\mu^{\prime}\right) \\
m_{Z} \sin \beta \sin \theta_{W} & -m_{Z} \sin \beta \cos \theta_{W} & -\left(\mu+\mu^{\prime}\right) & 0
\end{array}\right), \\
M_{\chi^{ \pm}}=\left(\begin{array}{cc}
M_{2} & \sqrt{2} m_{W} \sin \beta \\
\sqrt{2} m_{W} \cos \beta & \mu+\mu^{\prime}
\end{array}\right),
\end{gathered}
$$

where $M_{1}$ and $M_{2}$ are the $\mathrm{U}(1)$ and the $\mathrm{SU}(2)$ soft gaugino masses, respectively.

Here, it is important to note that, at the tree level, the NH soft higgsino mass parameter $\mu^{\prime}$ does not contribute to the scalar potential and hence does not directly affect the mass of the SM-like Higgs boson. As a result, the issue of electroweak fine-tuning arising from the so-called 'little hierarchy problem' $[13,14]$ gets somewhat decoupled from the effective higgsino mass given by $\mu+\mu^{\prime}$ [46]. Consequently, one could have a heavy higgsino LSP $(\sim 1 \mathrm{TeV})$ (which could be a viable DM candidate) without compromising on 'naturalness' when $\mu^{\prime}$ is large enough.

As pointed out in the Introduction, another significant phenomenological implication of the $\mathrm{NH}$ soft terms is a possible modification in the running of the fermion mass (Yukawa coupling), in particular, that of the bottom quark. This is primarily driven by $A_{b}^{\prime}$ and further assisted by an enhanced $\tan \beta$. At the one-loop level, the principal MSSM contributions to the bottom quark mass $m_{b}$ arise from the $\tilde{g}-\tilde{b}$ loop and the $\chi^{ \pm}-\tilde{t}$ loop diagrams as shown in figures 1(a) and 1(b) [65-68]. The trilinear soft interaction corresponding to the vertex $H_{u}-\tilde{t}_{R}-\tilde{t}_{L}$ contributes to the chargino loop via a factor $A_{t} y_{t} v_{u}$ where $v_{u}=\left\langle H_{u}\right\rangle$. 


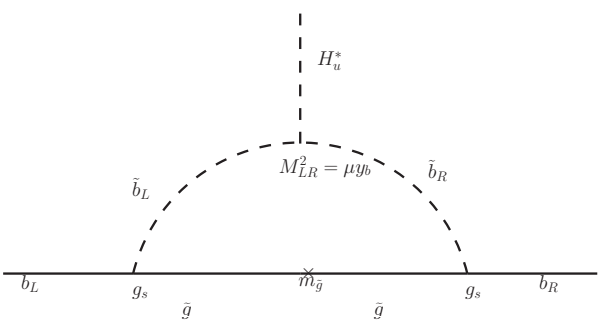

(a)

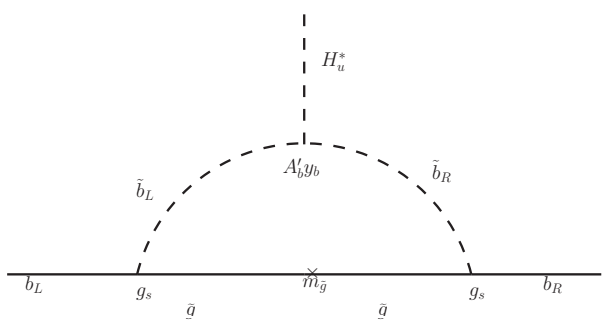

(c)

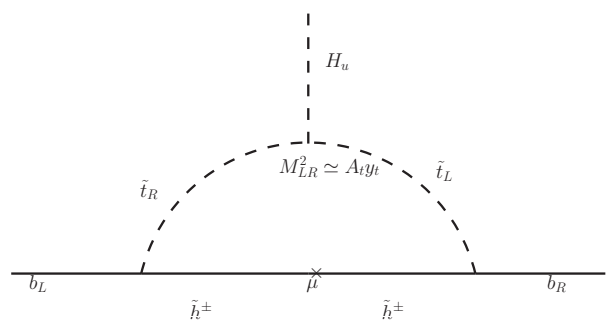

(b)

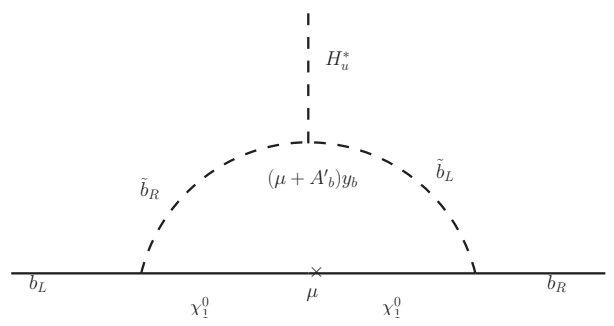

(d)

Figure 1. Principal one-loop diagrams for $\Delta m_{b}$ in the MSSM ((a) and (b)) and the NHSSM ((c) and (d)).

The two $q-\tilde{q}-\tilde{H}$ vertices in this one loop diagram that come from the superpotential interactions provide with a combined factor of $y_{t} y_{b}$. For the $\tilde{\chi}^{0}-\tilde{b}$ loop contribution to $m_{b}$ in the MSSM, one can have a trilinear soft interaction vertex factor $A_{b} y_{b} v_{d}$ in addition to the factor of $y_{b}^{2}$ contributed by the superpotential terms at the other two vertices. Thus, the $\chi_{0}-\tilde{b}$ contribution in the MSSM is much smaller compared to the $\chi^{ \pm}-\tilde{t}$ loop contribution. The $\tilde{g}-\tilde{b}$ contribution in the MSSM involves a factor containing the strong coupling constant $\alpha_{s}$. This is in addition to the factor that goes as $\mu y_{b}$ at the $\tilde{b}_{L}-H_{u}^{*}-\tilde{b}_{R}$ vertex and arises from the $F$-term contribution to the scalar potential.

On the other hand, in the NHSSM where $H_{u}$ is associated with the down type squarks (and sleptons) in the trilinear soft interactions, one can have a $\tilde{g}-\tilde{b}$ contribution with $A_{b}^{\prime} y_{b}$ showing up at the $\tilde{b}_{L}-H_{u}^{*}-\tilde{b}_{R}$ vertex (figure $1(\mathrm{c})$ ). Besides, there is a $\chi_{0}-\tilde{b}$ loop contribution (figure 1(d)) with the same factor of $A_{b}^{\prime} y_{b}$ at the trilinear scalar vertex and a factor of $y_{b}^{2}$ coming from the other two vertices with their origins in the superpotential. In addition, we will also take into account the MSSM contribution originating from the trilinear vertex involving the squarks and the Higgs boson that arises from the $F$-term of the scalar potential.

The relevant loop contributions to $m_{b}$ that we are concerned about in this work are those which are $\tan \beta$-enhanced. For the MSSM case, these come from $\tilde{g}-\tilde{b}$ and $\chi^{ \pm}-\tilde{t}$ loops and are given by $[65-70]$

$$
\begin{aligned}
\Delta m_{b}^{(\tilde{g})}{ }_{\text {MSSM }} & =\frac{2 \alpha_{3}}{3 \pi} m_{\tilde{g}} \mu y_{b} \frac{v_{u}}{\sqrt{2}} I\left(m_{\tilde{b}_{1}}^{2}, m_{\tilde{b}_{2}}^{2}, m_{\tilde{g}}^{2}\right), \\
\Delta m_{b}^{\tilde{h}^{+}}{ }_{\text {MSSM }} & =\frac{y_{t} y_{b}}{16 \pi^{2}} \mu A_{t} y_{t} \frac{v_{u}}{\sqrt{2}} I\left(m_{\tilde{t}_{1}}^{2}, m_{\tilde{t}_{2}}^{2}, \mu^{2}\right),
\end{aligned}
$$


where the loop integral $I(a, b, c)$ is given by

$$
I(a, b, c)=-\frac{a b \ln (a / b)+b c \ln (b / c)+c a \ln (c / a)}{(a-b)(b-c)(c-a)} .
$$

For the NHSSM, such contributions come from $\tilde{g}-\tilde{b}$ and $\chi_{0}-\tilde{b}$ loops and are given as follows: ${ }^{3}$

$$
\begin{aligned}
\Delta m_{b}^{(\tilde{g})}(\tilde{N H S S M} & =\frac{2 \alpha_{3}}{3 \pi} m_{\tilde{g}} A_{b}^{\prime} y_{b} \frac{v_{u}}{\sqrt{2}} I\left(m_{\tilde{b}_{1}}^{2}, m_{\tilde{b}_{2}}^{2}, m_{\tilde{g}}^{2}\right), \\
\Delta m_{b \text { NHSSM }}^{\tilde{h}^{0}} & =\frac{y_{b}^{2}}{16 \pi^{2}} \mu\left(\mu+A_{b}^{\prime}\right) y_{b} \frac{v_{u}}{\sqrt{2}} I\left(m_{\tilde{b}_{1}}^{2}, m_{\tilde{b}_{2}}^{2}, \mu^{2}\right) .
\end{aligned}
$$

Thus, for $\mu^{\prime}=0$ and for higgsino-dominated $\chi_{1}^{ \pm}$and LSP, we have the following (approximate) expression for $m_{b}$ where all the loop contributions are proportional to $\tan \beta$ :

$$
\begin{aligned}
m_{b} \approx \frac{y_{b} v_{d}}{\sqrt{2}}[1 & +\frac{y_{t}^{2}}{16 \pi^{2}} \mu A_{t} I\left(m_{\tilde{t}_{1}}^{2}, m_{\tilde{t}_{2}}^{2}, \mu^{2}\right) \tan \beta+\frac{2 \alpha_{3}}{3 \pi} m_{\tilde{g}}\left(\mu+A_{b}^{\prime}\right) I\left(m_{\tilde{b}_{1}}^{2}, m_{\tilde{b}_{2}}^{2}, m_{\tilde{g}}^{2}\right) \tan \beta \\
& \left.+\frac{y_{b}^{2}}{16 \pi^{2}} \mu\left(\mu+A_{b}^{\prime}\right) I\left(m_{\tilde{b}_{1}}^{2}, m_{\tilde{b}_{2}}^{2}, \mu^{2}\right) \tan \beta\right] .
\end{aligned}
$$

For a higgsino-dominated lighter chargino and the LSP, and for non-vanishing $\mu^{\prime}$, the loop function $I(a, b, c)$ will take $\left(\mu+\mu^{\prime}\right)^{2}$ in its argument in the NHSSM, instead of $\mu^{2}$.

\section{Sbottom phenomenology in the NHSSM (vis-a-vis the MSSM)}

As discussed earlier, the couplings involving the physical sbottom states and the electroweakinos are of particular importance in the study of how the NHSSM scenario could behave differently from the MSSM scenario. We thus first write down the expressions for the tree-level couplings involving these states. As can be seen, these couplings are functions of the elements of the matrices that diagonalize the mass (mass-squared) matrices in the electroweakino (scalar) sectors. As for the electroweakinos, the issue is straight-forward, i.e., these elements are mostly governed by $\mu_{\mathrm{eff}}=\mu+\mu^{\prime}$ and the gaugino mass parameters like $M_{1}$ and $M_{2}$. On the other hand, for the sbottom (and stop, as well) sector the elements of the diagonalizing matrix are functions of the nonholomorphic parameter $A_{b}^{\prime}\left(A_{t}^{\prime}\right)$ along with the standard MSSM parameters like $A_{b},{ }^{\prime} \mu$ ' and $\tan \beta$.

It needs to be pointed out here that, given the way $\mu^{\prime}$ enters the mass matrices in the electroweakino sector at the tree level (see equations (2.3) and (2.4)), it would be difficult to decipher an NHSSM effect just by studying the charginos and/or the neutralinos in isolation. Structurally, the same is true when dealing with the sbottom sector, given the way $A_{b}^{\prime}$ appears in the mass-squared matrix. When considered exclusively, both sectors could be viewed as though endowed with an effective value of ' $\mu$ ' given by $\mu+\mu$ ' for the electroweakino sector and $\mu+A_{b}^{\prime}$ for the sbottom sector, respectively. On their own, the mass-matrices would then mimic their MSSM counterparts. However, any study of sbottoms at the

\footnotetext{
${ }^{3}$ A detailed analysis for the effective Higgs vertices involving nonholomorphic $A^{\prime}$ terms with SUSY QCD corrections and electroweak effects can be found in references [71-73].
} 
colliders would inevitably involve their eventual decays to the LSP and possibly, to other lighter electroweakinos. It thus appears that definite imprints of the NHSSM could only be carried by the direct interactions of the sbottoms with the electroweakinos.

\subsection{Interactions with electroweakinos}

With the decay processes $\tilde{b}_{i} \rightarrow b \chi_{1}^{0}$ and $\tilde{b}_{i} \rightarrow t \chi_{1}^{-}$in reference where $i=1(2)$ refer to the lighter (heavier) mass eigenstates of the sbottom, their transition matrix elements involve the couplings $\tilde{b}_{i}-b-\chi_{1}^{0}$ and $\tilde{b}_{i}-t-\chi_{1}^{-}$, respectively. These contain a generic factor like $C_{L} P_{L}+C_{R} P_{R}$, where $P_{L, R}=\frac{1 \mp \gamma_{5}}{2}$ are the usual projection operators and $C_{L}$ and $C_{R}$ for the two processes are given by [74-76]

- for $\tilde{b}_{i}-b-\tilde{\chi}_{1}^{0}$ coupling:

$$
\begin{aligned}
& C_{L}=-\frac{i}{6}\left(-3 \sqrt{2} g_{2} N_{12}^{*} Z_{i 3}^{d}+6 N_{13} y_{b} Z_{i 6}^{d}+\sqrt{2} g_{1} N_{11} Z_{i 3}^{d}\right), \\
& C_{R}=-\frac{i}{3}\left(3 y_{b} Z_{i 3}^{d} N_{13}+\sqrt{2} g_{1} Z_{i 6}^{d} N_{11}\right),
\end{aligned}
$$

- for $\tilde{b}_{i}-t-\tilde{\chi}_{1}^{-}$coupling:

$$
\begin{aligned}
& C_{L}=i\left(y_{t} Z_{i 3}^{d} V_{12}\right), \\
& C_{R}=i\left(-g_{2} U_{11}^{*} Z_{i 3}^{d}+U_{12}^{*} y_{b} Z_{i 6}^{d}\right) .
\end{aligned}
$$

In the above expressions, $N_{i j}$ are the elements of the symmetric $(4 \times 4)$ matrix that diagonalizes the neutralino mass matrix of the same dimension in the basis $\left(\widetilde{B}, \widetilde{W}, \widetilde{H}_{d}^{0}, \widetilde{H}_{u}^{0}\right) . U_{i j}$ and $V_{i j}$ are the two unitary matrices that diagonalize the $(2 \times 2)$ asymmetric chargino mass matrix in the basis of charged wino and charged higgsino states. $Z_{i 3}^{d}$ and $Z_{i 6}^{d}$ are the elements of the unitary matrix that diagonalizes the mass-squared matrix for the down-type quark that give the left- and right-sbottom admixtures respectively, in the ' $i$ '-th sbottom mass-eigenstate. $g_{1}$ and $g_{2}$ are the usual $\mathrm{SU}(2)$ and $\mathrm{U}(1)$ gauge couplings, respectively, while $y_{b}$ and $y_{t}$ are the bottom and the top Yukawa couplings, respectively. For all these, we follow the convention and the notations adopted in the SARAH (v4.10.2) $[75,76]$ and SPheno (v4.0.3) [77] packages which take $0 \leq \theta_{\tilde{b}} \leq \pi$. Also, if $m_{L L}^{2}<m_{R R}^{2}, \cos \theta_{\tilde{b}}>\frac{1}{\sqrt{2}}$ and $\tilde{b}_{1}$ would have a dominant $\tilde{b}_{L}$ admixture where $m_{L L}\left(m_{R R}\right)$ is the diagonal entry in equation (2.2) for the left (right) sectors, respectively. The converse is true as well.

At this point, it is important to note that the coupling of a sbottom state to a higgsinolike neutralino is always proportional to $y_{b}$ while the same to a top quark and a higgsinolike chargino depends on the chiral admixture it possesses. Such a coupling for a left-like sbottom is governed by $y_{t}$ while that for a right-like sbottom goes as $y_{b}$. Hence, clearly, when allowed by phase space, a left-like sbottom dominantly decays to $t \chi_{1}^{-}$thus leading to a small branching fraction for the $b \chi_{1,2}^{0}$ final state when $\chi_{1,2}^{0}$ are both higgsino-dominated and light. This is no different from the MSSM. However, in the NHSSM scenario, the presence of a non-vanishing $A_{b}^{\prime}$ alters the composition of the sbottom states in a nontrivial way. This could lead to different observable effects when compared to the MSSM case. 


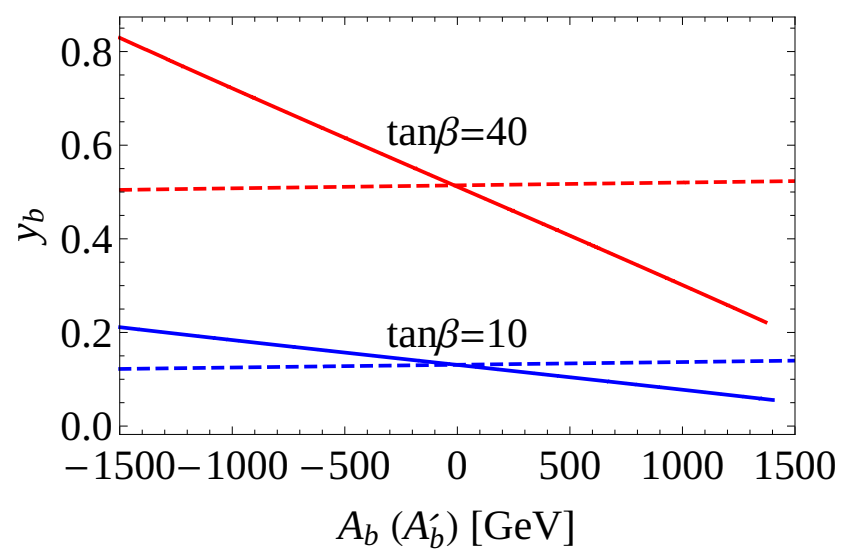

Figure 2. Variation of $y_{b}$ as a function of $A_{b}^{\prime}$ (in the NHSSM with $A_{b}=0$; bold lines) and $A_{b}$ (in the MSSM; broken lines) for $\tan \beta=10$ (in blue) and for $\tan \beta=40$ (in red). Some of the fixed input parameters are $\mu=200 \mathrm{GeV}, \mu^{\prime}=0, M_{1}=500 \mathrm{GeV}$ and $M_{2}=1.1 \mathrm{TeV}$ while the rest are presented in table 1.

Note that there may be another competing decay mode of an sbottom state in the form of $\tilde{b}_{1} \rightarrow \tilde{t}_{1} W^{-}$which is often considered in a detailed study of the sbottom sector. In the present work, however, we restrict ourselves to a simpler situation where this decay is kinematically forbidden.

Interestingly, the couplings mentioned above receive nontrivial contributions through $y_{b}$. The effects here are of two types: first, $y_{b}$ has the usual dependence on $\tan \beta$ as in the MSSM case [65-70]. More crucially, in the NHSSM scenario, $y_{b}$ becomes a function of $A_{b}^{\prime}$ via radiative corrections to $m_{b}$. The dependence is studied via the SARAH-generated SPheno implementation of the NHSSM scenario The variation is illustrated in figure 2 for two values of $\tan \beta$. The curves reveal usual dependence (direct proportionality) of $y_{b}$ on $\tan \beta$. In addition, significantly enough, the variation of $y_{b}$ with $A_{b}^{\prime}$ is found to be rather large (up to 4 times for the range of $A_{b}^{\prime}$ we consider). As can be gleaned from figure 2 , the larger values of $y_{b}$ are obtained for larger $\tan \beta$ and for $A_{b}^{\prime}<0$. In contrast, the variation of $y_{b}$ as a function of $A_{b}$ in the MSSM is known to be much smaller in comparison. These are shown in broken lines for the two values of $\tan \beta$ under consideration. It is also to be noted that, for a given $\tan \beta$, NHSSM leads to larger $y_{b}$ values when compared to the MSSM case only if $A_{b}^{\prime}<0$. The reverse is the case when $A_{b}^{\prime}>0$.

We now move on to study the dependence of the effective strengths of the vertices $\tilde{b}_{1}-b-\chi_{1}^{0}$ and $\tilde{b}_{1}-t-\chi_{1}^{-}$that appear in the decays $\tilde{b}_{1} \rightarrow b \chi_{1}^{0}$ and $\tilde{b}_{1} \rightarrow t \chi_{1}^{-}$, respectively, on $A_{b}^{\prime}$. This assumes that pair-production of $\tilde{b}_{1}$ has the dominant contribution to the final state we would be interested in. However, in section 3.3.2 we would take a careful look as to how significant the contributions from $\tilde{b}_{2}$ pair-production could turn out to be.

At the level of squared matrix elements for these decays, the interaction strengths are constituted of appropriate $C_{L}$ 's and $C_{R}$ 's of equations (3.1) and (3.2). We have checked analytically that squared matrix-elements for these decays dominantly depend on the factor $C_{L}^{2}+C_{R}^{2}$. We thus study the variations of this factor as functions of $A_{b}^{\prime}$ and $\tan \beta$ for the two decay processes mentioned above and for two representative scenarios: one with a higgsino- 

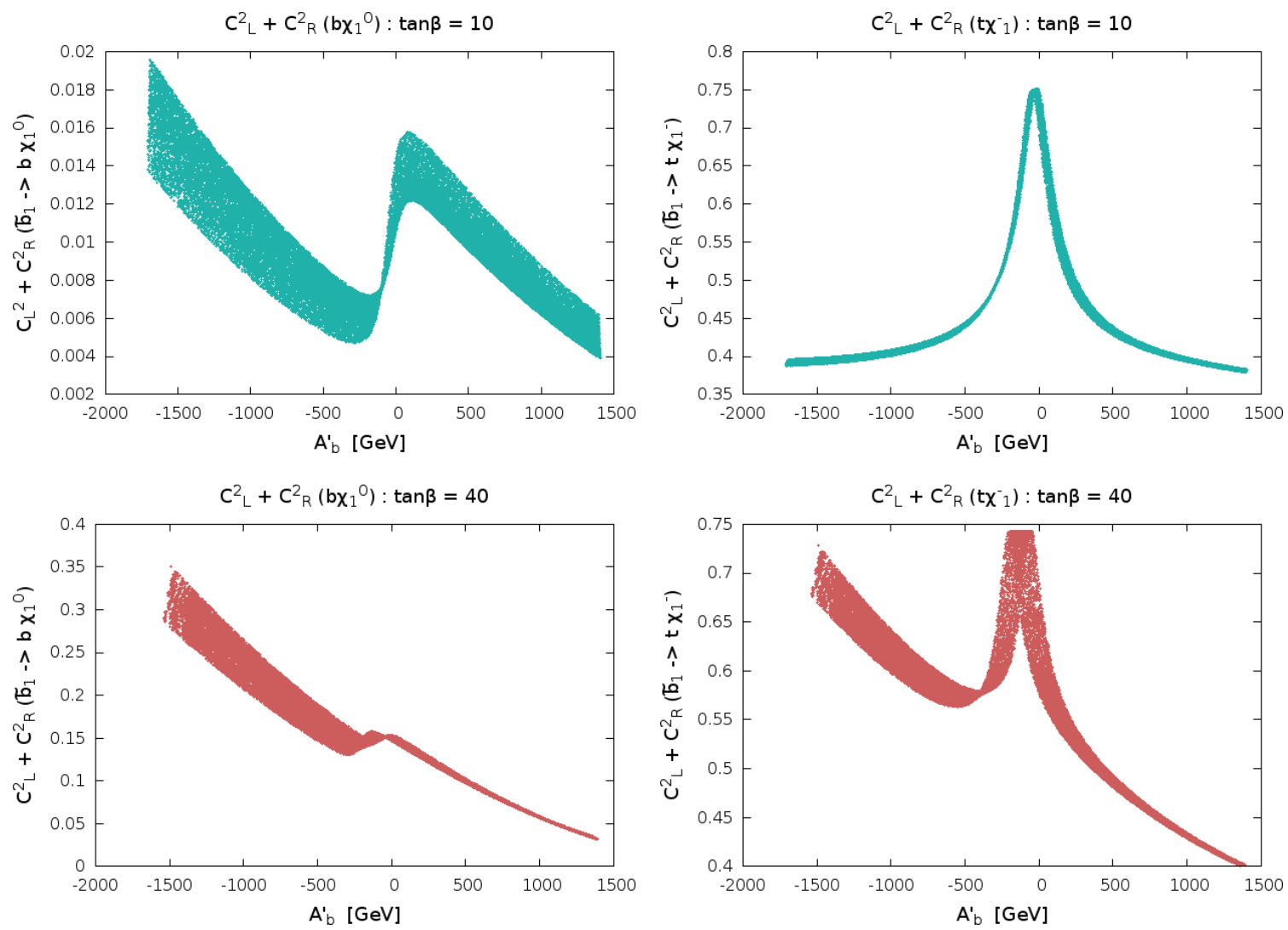

Figure 3. Variation of the effective interaction strengths $\left(C_{L}^{2}+C_{R}^{2}\right)$ involved in the decays $\tilde{b}_{1} \rightarrow b \chi_{1}^{0}$ (left) and $\tilde{b}_{1} \rightarrow t \chi_{1}^{-}$(right) as functions of $A_{b}^{\prime}$ for $\tan \beta=10$ (top) and for $\tan \beta=40$ (bottom) in a scenario with a higgsino-like neutralino LSP. The fixed input range of ' $\mu$ ' is $100 \mathrm{GeV} \leq \mu \leq 350 \mathrm{GeV}$. Other fixed parameters used are as follows: $M_{1}=500 \mathrm{GeV}, M_{2}=1.1 \mathrm{TeV}, \mu^{\prime}=0, A_{b}=0$ while $m_{\tilde{b}_{L}}=m_{\tilde{b}_{R}}=1.2 \mathrm{TeV}$.

like neutralino LSP (figure 3 ) and the other with a gaugino (bino)-like neutralino LSP (figure 4 ). The values of $C_{L}$ and $C_{R}$ for the two decay processes are obtained from SARAH. In both figures, plots on left (right) stand for the effective strength of the vertex $\tilde{b}_{1}-b-\chi_{1}^{0}$ $\left(\tilde{b}_{1}-t-\chi_{1}^{-}\right)$and the top panels correspond to an input $\tan \beta$ value of 10 . However, the bottom panel in figure 3 (figure 4) corresponds to $\tan \beta=40$ (20). The compulsion for choosing two different values of 'higher' $\tan \beta$ (40 versus 20 ) to demonstrate the said variation in the scenarios with higgsino- and gaugino-like LSP is elaborated later in section 3.2, in an appropriate context.

It is clear from figure 3 that, for both modes of decay, the involved interaction strengths vary significantly with $A_{b}^{\prime}$. The bands arise since ' $\mu$ ' is varied (over the range $100 \mathrm{GeV}$ $\leq \mu \leq 350 \mathrm{GeV}$ ) which, all through, keeps the LSP dominantly higgsino-like) for our choices of $M_{1}$ and $M_{2}$. For the $\tilde{b}_{1}-b$ - $\chi_{1}^{0}$ vertex, the effective interaction strength could vary by a factor as large as 5 (10) for $\tan \beta=10$ (40). For the $\tilde{b}_{1}-t-\chi_{1}^{-}$vertex, the corresponding variation is found to be by a factor of 2 , approximately, irrespective of $\tan \beta$. Also, the generic strength of the effective $\tilde{b}_{1}-b-\chi_{1}^{0}$ interaction is much smaller than that for $\tilde{b}_{1}-t-\chi_{1}^{-}$. 

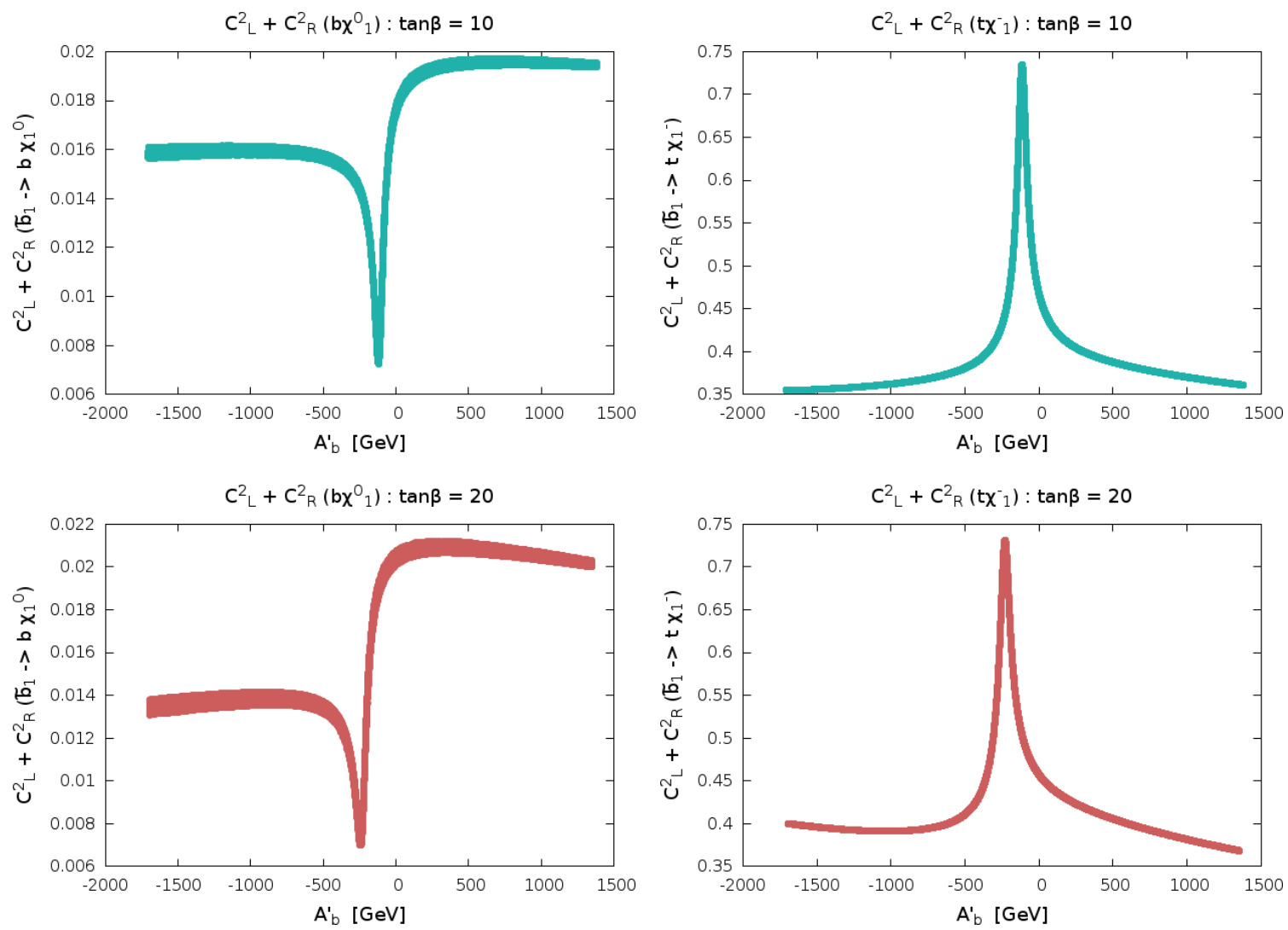

Figure 4. Same as in figure 3 but for a scenario having a gaugino (bino)-like LSP with $\mu=900 \mathrm{GeV}$ while the fixed input range of $M_{1}$ used is $100 \mathrm{GeV} \leq M_{1} \leq 350 \mathrm{GeV}$. Also, the larger of the two $\tan \beta$ values used here is 20 (bottom panel) instead of 40 used in figure 3 (see text for details).

This is mainly because the latter is governed by $y_{t}$ when $\tilde{b}_{1} \sim \tilde{b}_{L}$, which happens to be the case here.

The profiles in figure 3 can also be understood in the following terms. For the $\tilde{b}_{1}$ $b-\chi_{1}^{0}$ interaction (plots on the left), the LSP being dominantly a higgsino, the coupling factor $C_{L}^{2}+C_{R}^{2}$ is mostly determined by $y_{b}$. Hence its variations with $A_{b}^{\prime}$ exhibit a nature similar to the corresponding variations of $y_{b}$. Dips appear at $A_{b}^{\prime}$ values with commensurate magnitudes of ' $\mu$ ' for which $\mu+A_{b}^{\prime} \simeq 0$. This flags a situation with the least chiralmixing between the sbottom states thus rendering the $\tilde{b}_{1}$ state to be almost purely lefthanded. Thereon, an increase in $A_{b}^{\prime}$, as it changes its sign to positive, flips the sign of $\cos \theta_{\tilde{b}}=Z_{1,3}^{d}$ but the latter still (nearly) retaining the magnitude it had for $A_{b}^{\prime}<0$. This results in shooting up of the interaction strength as the sign on $A_{b}^{\prime}$ flips from negative to positive. Further increase in $A_{b}^{\prime}$ leads to a monotonic drop in the interaction strength which merely follows the trend $y_{b}$ exhibits as a function of $A_{b}^{\prime}$. We will see later (see figure 4) that this feature gets reflected in the variation of the branching ratio $\operatorname{BR}\left[\tilde{b}_{1} \rightarrow b \chi_{1}^{0}\right]$ as a function of $A_{b}^{\prime}$.

For the $\tilde{b}_{1}-t-\chi_{1}^{-}$interaction (plots on the right) issues are a little more involved. As noted earlier in this section, in the central region, with $\tilde{b}_{1} \sim \tilde{b}_{L}$, the interaction strength 
for $\tilde{b}_{1}-t-\chi_{1}^{-}$preferentially depends on $y_{t}$. This effect is reflected in the peaks in the center of these plots. Further, the bigger the magnitude of $A_{b}^{\prime}$ is, the larger is the admixture of $\tilde{b}_{R}$ in $\tilde{b}_{1}$ which, in turn, makes the interaction increasingly more dependent on $y_{b}$. However, for $\tan \beta$ not so large $(=10)$, as is the case in the top right plot, even for large negative $A_{b}^{\prime}$, $y_{b}$ cannot compete with $y_{t}$ that shapes the peak around $A_{b}^{\prime}=0$. In contrast, in the bottom right plot, for which $\tan \beta$ is much larger $(=40), y_{b}$ is expectedly much enhanced for large negative $A_{b}^{\prime}$ (see figure 2). This drives the interaction strength up to the level obtained for the $y_{t}$-driven regime of the central region with small $A_{b}^{\prime}$. This plot also corroborates with figure 2 that $y_{b}$ decreases steadily with increasing positive value of $A_{b}^{\prime}$. The bands appear due to a variation of ' $\mu$ ' over the range indicated. while the central peak arises from $y_{t}$-dominance. Overall, it may be noted that the effective strength is small for the $\tilde{b}_{1}-b$ - $\chi_{1}^{0}$ interaction when compared to the one for the $\tilde{b}_{1}-t-\chi_{1}^{-}$interaction. In fact, for $\tan \beta=10$, the former always remains much smaller.

Descriptions of the plots in figure 4 follow a similar line of argument but for the fact that the LSP is now bino-dominated. Thus, the $\tilde{b}_{1}-b-\chi_{1}^{0}$ coupling is driven by the hypercharge of the sbottom. Hence the interaction strength would be higher for $\tilde{b}_{1}$ with a larger admixture of $\tilde{b}_{R}$. This is clearly reflected in the plots on the left where dips appear at the central region with smaller $\left|A_{b}^{\prime}\right|$ for which the $\tilde{b}_{1}$ is $\tilde{b}_{L}$-dominated. With an increasing magnitude of $A_{b}^{\prime}$, $\tilde{b}_{R}$ admixture grows quickly to a maximum (see the $\cos \theta$ variation plot of figures 7 and 8 .) and the effect of this is reflected in the wings of these curves that take off fast and then spread out flat for larger values of $\left|A_{b}^{\prime}\right|$. On the other hand, plots on the right representing the $\tilde{b}_{1}-t-\chi_{1}^{-}$case (in particular, the top right one with $\tan \beta=10$ ) are (is) rather similar to the corresponding ones (one) in figure 3 since the lighter chargino still remains to be dominantly higgsino-like, though heavier, as $m_{\chi_{1}^{ \pm}} \approx \mu=900 \mathrm{GeV}$ and $M_{1} \ll \mu \ll M_{2}$, with $M_{1}$ and $M_{2}$ set at $500 \mathrm{GeV}$ and $1.1 \mathrm{TeV}$, respectively. Some differences arise for the bottom right plot for larger magnitudes of $A_{b}^{\prime}$. This is since, as described earlier, in this region the $y_{b}$ effect dominates and, given that we are using a smaller value for $\tan \beta$ (20, versus 40 , in figure 3), $y_{b}$ is smaller in the present case, for a given $A_{b}^{\prime}$. Note further that the parts in the central region (showing the peaks) where $y_{t}$ governs are expectedly not affected much by this difference in $\tan \beta$. Again, as for the higgsino-dominated LSP scenario of figure 3, here also the strength of the $\tilde{b}_{1}-b-\chi_{1}^{0}$ interaction remains to be much smaller than that of the $\tilde{b}_{1}-t-\chi_{1}^{-}$interaction. Also, note that in contrast to figure 3 , even for the larger $\tan \beta$ $(=20)$ value considered in this figure, the $\tilde{b}_{1}-b-\chi_{1}^{0}$ coupling does not get enhanced since it dominantly involves gauge coupling $g_{1}$ and not $y_{b}$.

\subsection{Masses, mixings and decays of the lighter sbottom}

The branching fractions of $\tilde{b}_{1}$ to various competing modes are governed not only by the couplings involved but also by the phase space available for such decays. The latter, in turn, depends on the mass-splittings between $\tilde{b}_{1}$ and the sparticle it decays to, of which the important ones are the electroweakinos (a neutralino or a chargino) and, possibly, the lighter stop state $\left(\tilde{t}_{1}\right)$. As mentioned earlier, in the present study, decays of $\tilde{b}_{1}$ to electroweakinos would play more direct roles though. Hence, for simplicity, we consider $m_{\tilde{b}_{1}}<m_{\tilde{t}_{1}}+m_{W}$ such that the decay $\tilde{b}_{1} \rightarrow \tilde{t}_{1} W^{-}$is kinematically forbidden. 

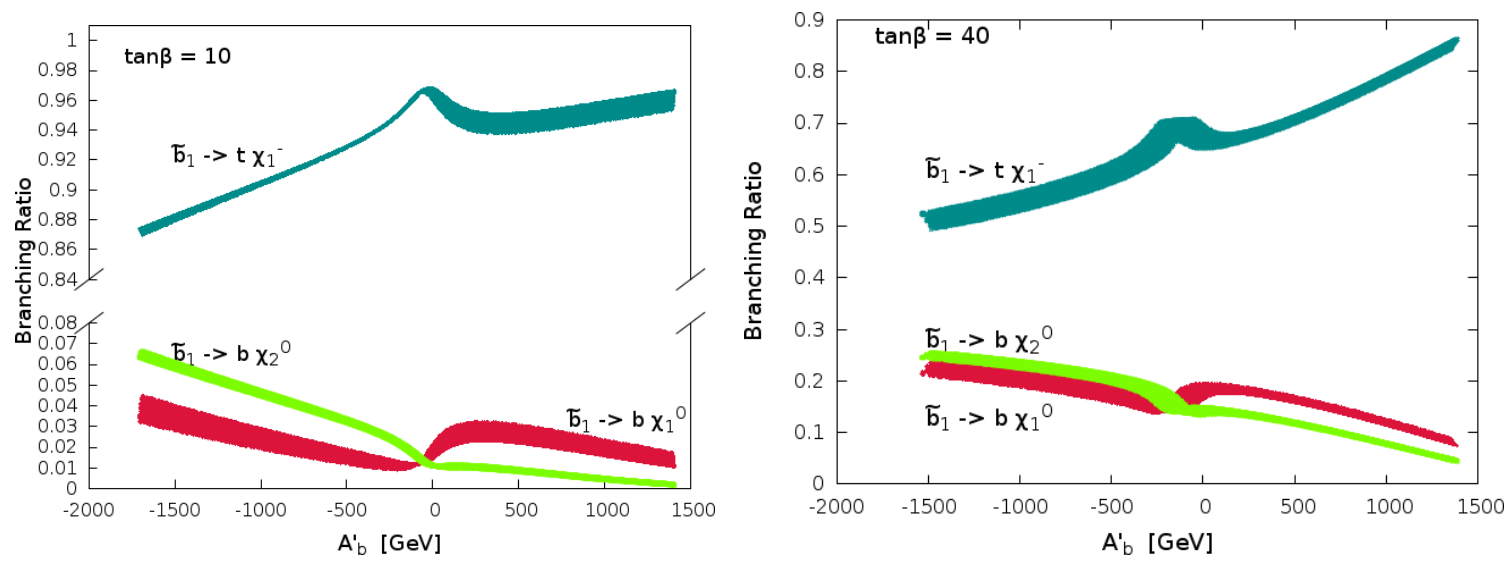

Figure 5. Variations of various decay branching fractions of $\tilde{b}_{1}$ as a function of $A_{b}^{\prime}$ when the LSP is higgsino-like for $\tan \beta=10$ (left) and $\tan \beta=40$ (right). Other fixed/varying input parameters are as in figure 3. A broken ordinate is chosen to present the branching ratio in the plot on the left.
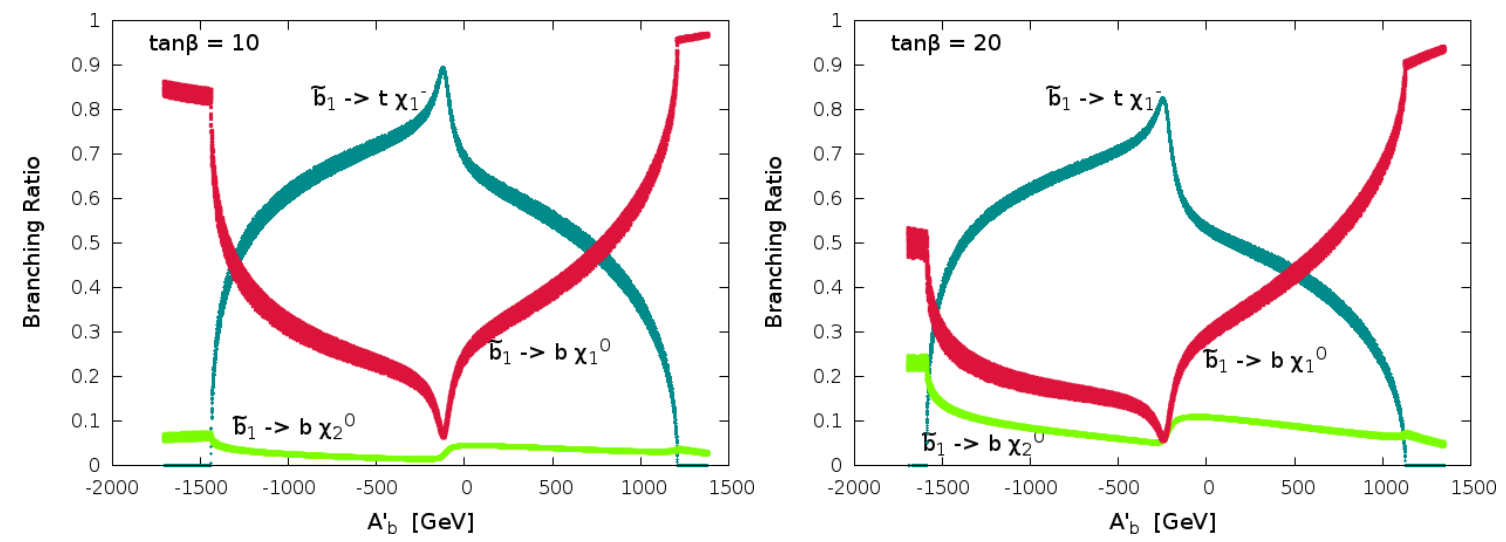

Figure 6. Same as in figure 5 but for a bino-like LSP. Other fixed/varying parameters are as in figure 4 .

We discuss two representative scenarios in this context. In the first scenario, $\mu_{\mathrm{eff}}$ remains small and is much smaller than $M_{1,2}$. This renders $\chi_{1}^{0}, \chi_{2}^{0}$ and $\chi_{1}^{ \pm}$higgsino-like. In the second one, $M_{1} \ll \mu_{\text {eff }} \ll M_{2}$ leading to a bino-like neutralino LSP while $\chi_{1}^{ \pm}$is still higgsino-like but could be much heavier than the LSP. In figures 5 and 6 we present the variations of the branching fractions of $\tilde{b}_{1}$ as functions of $A_{b}^{\prime}$ for these two scenarios, respectively. In both these figures, plots on the left (right) correspond to $\tan \beta=10$ (40). We find that the patterns of variation are very different for the two scenarios. On a closer look, we find that these derive from those for the corresponding interaction strengths as depicted in figures 3 and 4 . This can also be understood in the following way. The interaction strength for $\tilde{b}_{1}-t-\chi_{1}^{-}$is generally larger than that for $\tilde{b}_{1}-b-\chi_{1}^{0}$, the difference getting smaller with increasing $y_{b}$ (i.e., at large negative $A_{b}^{\prime}$ and large $\tan \beta$ ). Hence the total decay width appearing in the denominator of the formula for branching fractions is dominated by the width of $\tilde{b}_{1} \rightarrow t \chi_{1}^{-}$and competes with $\tilde{b}_{1} \rightarrow b \chi_{1,2}^{0}$ only in the region where 
$y_{b}$ is enhanced. In the regions where the interaction strengths for $\tilde{b}_{1} \rightarrow t \chi_{1}^{-}$have flatter profiles (e.g., for $\tan \beta=10$ and larger $\left|A_{b}^{\prime}\right|$ ), the variation of $\mathrm{BR}\left[\tilde{b}_{1} \rightarrow b \chi_{1}^{0}\right]$ would follow the same for its decay width which, in turn, goes as the corresponding interaction strength. For smaller $\left|A_{b}^{\prime}\right|$ where the change of the width for $\tilde{b}_{1} \rightarrow t \chi_{1}^{-}$is somewhat abrupt, the variation of $\mathrm{BR}\left[\tilde{b}_{1} \rightarrow b \chi_{1}^{0}\right]$ still broadly follows the profile of the involved coupling strength. This is more or less true for both the cases with higgsino- and gaugino-dominated LSP. The region where $y_{b}$ becomes large (e.g., for $\tan \beta=40$ and larger $\left|A_{b}^{\prime}\right|$ ) is only relevant when the LSP (lighter chargino) is higgsino-dominated for which a competition among the said decay modes set in and the profiles for the branching ratios shape up accordingly.

We further need the information as to how $m_{\tilde{b}_{1}}$ varies as a function of $A_{b}^{\prime}\left(A_{b}\right.$, in the MSSM). For simplicity, this can be studied for a fixed set of values of the sbottom soft masses $\left(m_{\tilde{b}_{L}}\right.$ and $\left.m_{\tilde{b}_{R}}\right){ }^{4}$ Intimately connected with this variation is the chiral content of $\tilde{b}_{1}$ which, in turn, dictates the strength of its various interactions. Hence presenting simultaneous variations of these factors would perhaps be the best means to understand their interplay. In figures 7 and 8 we illustrate these variations for the cases with a higgsinolike and a gaugino (bino)-like neutralino LSP, respectively.

In both figures 7 and 8 , the top panel presents the case for $\tan \beta=10$. However, for the bottom panel $\tan \beta$ is set to 40 (20) for figure 7 (figure 8). The reason behind this is that the larger value of $\mu(=900 \mathrm{GeV})$ used to obtain a gaugino-like neutralino LSP in the latter case hardly finds a situation with $\tan \beta \gtrsim 20$ and still yielding an acceptable (nontachyonic) spectrum of particles over the range of $A_{b}^{\prime}$ that ensures a substantial variation (drop) in $m_{\tilde{b}_{1}}$. Note that the plots in the top panel directly correspond to the same in figure 3 depicting the involved coupling strengths.

All plots are shown in the $A_{b}^{\prime}\left(A_{b}\right)-m_{\tilde{b}_{1}}$ plane. Thus, the common backdrop they present is the variation of $m_{\tilde{b}_{1}}$ as a function of $A_{b}^{\prime}\left(A_{b}\right)$ in the NHSSM (MSSM). The fixed value of the soft masses are taken to be $m_{\tilde{b}_{L}}=m_{\tilde{b}_{R}}=1.2 \mathrm{TeV}$. This is so chosen that, for the entire range of variation of other input parameters, $m_{\tilde{b}_{1}}$ always remains around $1 \mathrm{TeV}$ thus more than satisfying the most conservative bound from the LHC [80] on the same. The values/ranges we employed for all the relevant SUSY input parameters are presented in table 1. For figure 7 (figure 8) we choose $\mu=200$ (900) $\mathrm{GeV}$ while $M_{1}$ and $M_{2}$ are fixed at $500 \mathrm{GeV}$ and $1.1 \mathrm{TeV}$, respectively for both the figures.

For both figures 7 and 8 , we have made a simplifying choice of $\mu^{\prime}=A_{b}=0$ for the NHSSM case. ${ }^{5}$ For the MSSM case, $A_{b}$ is varied over the same range as for $A_{b}^{\prime}$ in the NHSSM case. Varying colors (from the adjacent palettes) indicate changing sbottom mixing angle (in terms of $\cos \theta_{\tilde{b}}$ ) for plots on the left of these figures and varying branching ratio (fraction) $\mathrm{BR}\left[\tilde{b}_{1} \rightarrow b \chi_{1}^{0}\right]$ for those on the right. Clearly, $\cos \theta_{\tilde{b}}$ ranges between $\frac{1}{\sqrt{2}} \approx 0.7$ (maximal mixing) and $1\left(\tilde{b}_{1} \equiv \tilde{b}_{L}\right.$ limit) signifying $\tilde{b}_{1}$ to be $\tilde{b}_{L}$-dominated. Flatter lines

\footnotetext{
${ }^{4}$ Throughout this work, we ensure that the parameter points are consistent with the current bounds on the Higgs sector as implemented in the packages HiggsBounds (v4.3.1) [78] and HiggsSignals (v1.4.0) [79].

${ }^{5}$ Since $\mu^{\prime}$ and $A_{b}^{\prime}$ exclusively affect only the electroweakino and the sbottom sectors, respectively (at least, at the tree-level), $A_{b}^{\prime}=0$ could appear to be a viable alternate choice. However, given that $y_{b}$ has a non-trivial dependence on $A_{b}^{\prime}$, here we choose to vary the latter. In section 3.3.1 (see figure 10), we would simultaneously vary $\mu^{\prime}$ and $A_{b}^{\prime}$ to study some observable effects.
} 

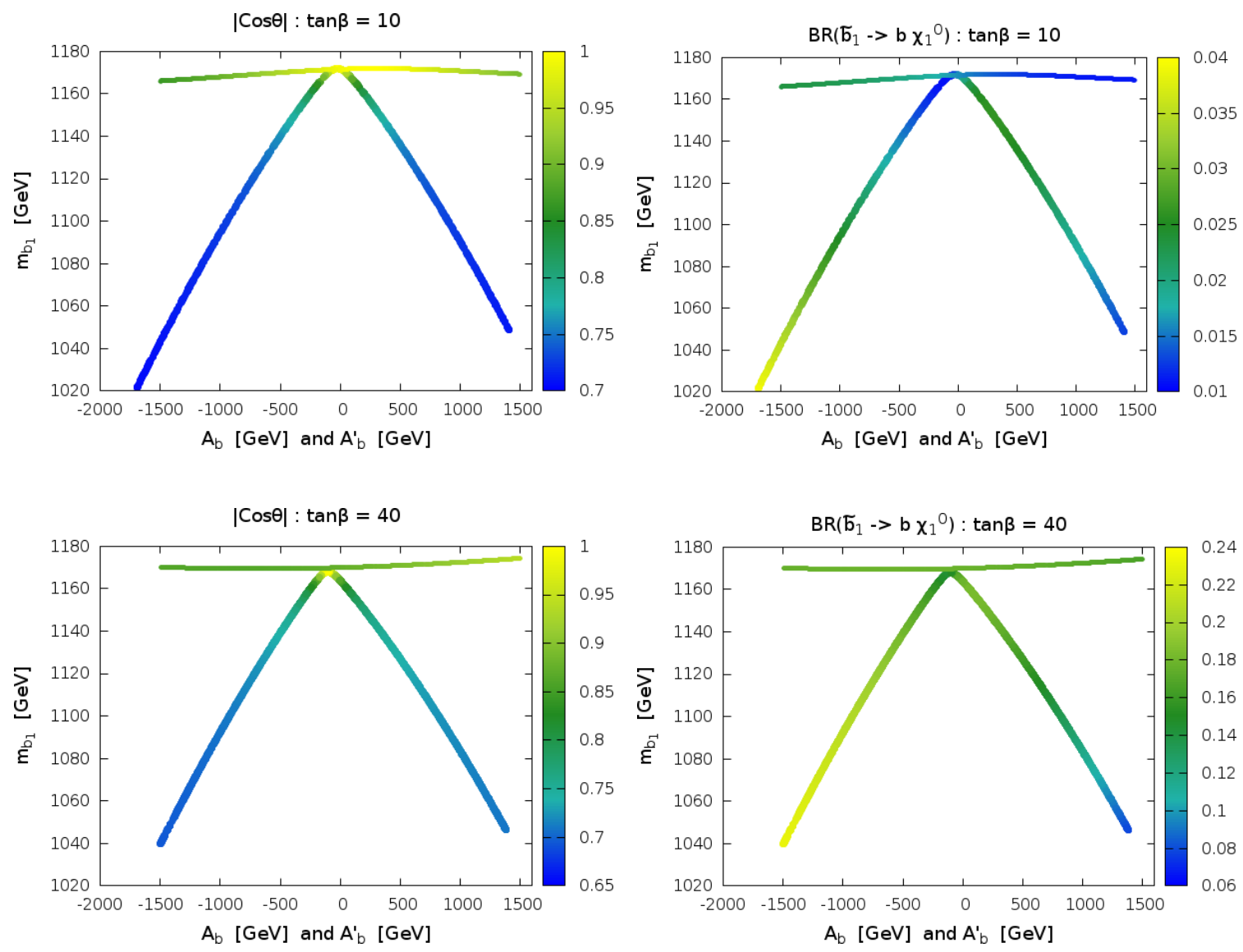

Figure 7. Variations of $\cos \theta_{\tilde{b}}$ (left) and $\mathrm{BR}\left[\tilde{b}_{1} \rightarrow b \chi_{1}^{0}\right]$ (right) in the $A_{b}^{\prime}-m_{\tilde{b}_{1}}$ plane for $\tan \beta=10(40)$ in the top (bottom) panel for a scenario with both the LSP neutralino and the lighter chargino being higgsino-like. The fixed parameters used are as follows: $\mu=200 \mathrm{GeV}, M_{1}=500 \mathrm{GeV}$, $M_{2}=1.1 \mathrm{TeV}, A_{b}=0, \mu^{\prime}=0, m_{\widetilde{Q}_{3}}=m_{\tilde{t}_{L}}=m_{\tilde{b}_{L}}=m_{\tilde{b}_{R}}\left(m_{\widetilde{D}_{3}}\right)=1.2 \mathrm{TeV}$ and $m_{\tilde{t}_{R}}\left(m_{\widetilde{U}_{3}}\right)=1.5 \mathrm{TeV}$. Flatter lines represent the corresponding variations as functions of $A_{b}$ in the MSSM.

at the top of these plots illustrate the corresponding variations in the MSSM for varying $A_{b}$ and are introduced to highlight the extent to which issues basic to the phenomenology (masses and mixing angles) could get altered in the NHSSM scenario.

Figures 7 and 8 reveal the following important information. These tell us that over the range of variation of $A_{b}^{\prime}$ shown, $m_{\tilde{b}_{1}}$ could vary by $\lesssim 160 \mathrm{GeV}$. It is a significant variation in view of the fact that the corresponding number in the MSSM (as a function of $A_{b}$, varied over the same range as $A_{b}^{\prime}$ ) reaches at most $20 \mathrm{GeV}$. This could be understood in the following way. It may appear that a comparable range of variation in $m_{\tilde{b}_{1}}$, in the MSSM, could be found just by allowing ' $\mu$ ' to vary over a larger range thereby compensating for the missing $A_{b}^{\prime}$. However, this is not correct. In fact, the major effect in this respect, in the NHSSM, does not come directly from $A_{b}^{\prime}$, per se, in the off-diagonal element of the masssquared matrix. Rather, a significant variation of $y_{b}$ with $A_{b}^{\prime}$, as illustrated in figure 2, induces such a big change in $m_{\tilde{b}_{1}}$. In addition, plots on the left clearly show that, in the NHSSM, with increasing magnitude of $A_{b}^{\prime}$, one quickly achieves a close-to-maximal mixing in the sbottom sector while the same is difficult to find in the MSSM, for a varying $A_{b}$. 

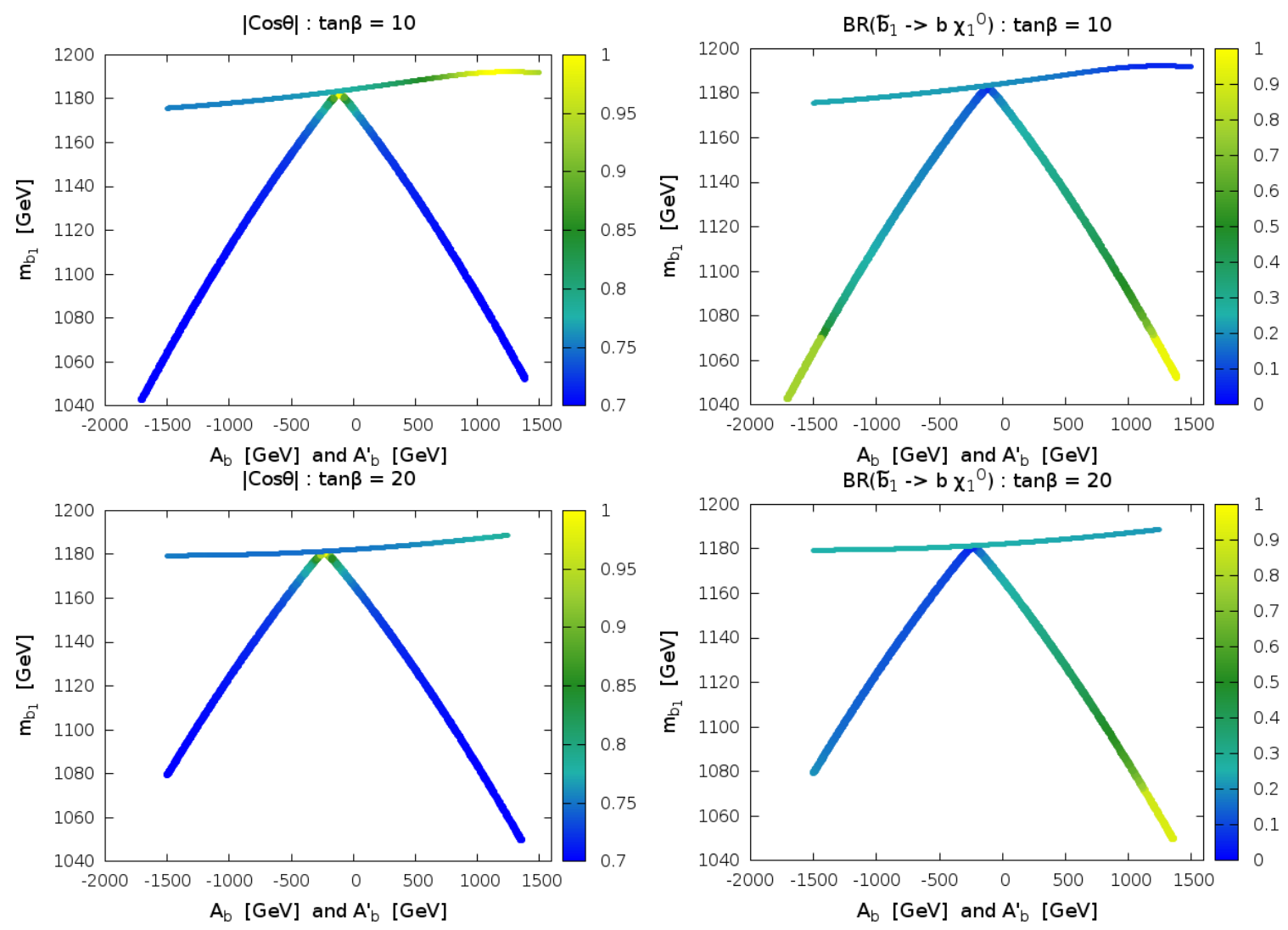

Figure 8. Same as in figure 7 but for a scenario with gaugino (bino)-like neutralino LSP which is ensured by setting $M_{1}=500 \mathrm{GeV}, M_{2}=1.1 \mathrm{TeV}$ and $\mu=900 \mathrm{GeV}$ while $\tan \beta=20$ for the plots in the bottom panel. The specific choice of ' $\mu$ ' also ensures that the decay mode $\tilde{b}_{1} \rightarrow t \chi_{1}^{-}$could be open or closed depending upon varying $m_{\tilde{b}_{1}}$ as a function of $A_{b}^{\prime}$.

Plots on the right of these figures depict the variation of $\operatorname{BR}\left[\tilde{b}_{1} \rightarrow b \chi_{1}^{0}\right]$. One finds that in a scenario with a higgsino-like LSP (figure 7 ), $\operatorname{BR}\left[\tilde{b}_{1} \rightarrow b \chi_{1}^{0}\right]$ is typically small and barely reaches $\sim 25 \%$ for large $\tan \beta$ values while, in the scenario with a gaugino (bino)like LSP (figure 8), the same could attain a value of $100 \%$. This is grossly a kinematic effect where the larger value of ' $\mu$ ' leads to a heavier $\chi_{1}^{ \pm}$thereby suppressing the competing $\mathrm{BR}\left[\tilde{b}_{1} \rightarrow t \chi_{1}^{-}\right]$. Also, note that larger values of $\mathrm{BR}\left[\tilde{b}_{1} \rightarrow b \chi_{1}^{0}\right]$ are obtained for $A_{b}^{\prime}<0(>0)$ for scenarios with a higgsino-like (gaugino-like) LSP. However, the reasons behind such enhancements in the two cases have a subtle difference. For the former, $\operatorname{BR}\left[\tilde{b}_{1} \rightarrow b \chi_{1}^{0}\right]$ gets directly reinforced due to an enhanced $y_{b}$ as $A_{b}^{\prime}<0$. For the latter, the enhanced $\operatorname{BR}\left[\tilde{b}_{1} \rightarrow b \chi_{1}^{0}\right]$ is due to closure of the decay $\tilde{b}_{1} \rightarrow t \chi_{1}^{-}$as $m_{\tilde{b}_{1}}$ drops to a critical level with

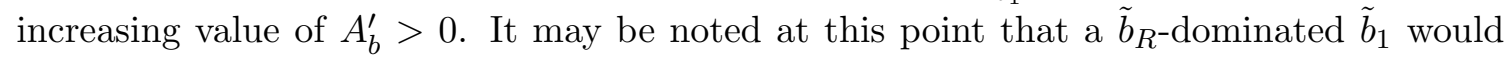
lead to an enhanced branching fraction to bottom quark and LSP for both the higgsinoand bino-dominated LSP. Thus, by sticking to $\tilde{b}_{1} \simeq \tilde{b}_{L}$, we made a conservative choice as far as the final-state yields are concerned. 


\begin{tabular}{|c|c|c|}
\hline Parameters & MSSM $(\mathrm{GeV})$ & NHSSM $(\mathrm{GeV})$ \\
\hline$M_{2,3}$ & \multicolumn{2}{|c|}{1100,2800} \\
\hline$m_{\tilde{Q}_{1,2}} / m_{\tilde{D}_{1,2}} / m_{\tilde{U}_{1,2}}$ & \multicolumn{2}{|c|}{2000} \\
\hline$m_{\tilde{Q}_{3}}, m_{\tilde{D}_{3}}, m_{\tilde{U}_{3}}$ & \multicolumn{2}{|c|}{$1200,1200,1500$} \\
\hline$m_{\tilde{L}_{1,2}} / m_{\tilde{E}_{1,2}}$ & \multicolumn{2}{|c|}{1800} \\
\hline$m_{\tilde{L}_{3}} / m_{\tilde{E}_{3}}$ & \multicolumn{2}{|c|}{2000} \\
\hline$m_{A}$ & \multicolumn{2}{|c|}{2000} \\
\hline $\tan \beta$ & \multicolumn{2}{|c|}{10} \\
\hline$A_{t}, A_{\tau}$ & \multicolumn{2}{|c|}{2000,0} \\
\hline$A_{b}$ & {$[-1500: 1500]$} & 0 \\
\hline \multirow{3}{*}{$\begin{array}{c}A_{b}^{\prime} \\
A_{t}^{\prime}, A_{\mu}^{\prime}, A_{\tau}^{\prime} \\
\mu^{\prime}\end{array}$} & - & {$[-1500: 1500]$} \\
\hline & - & $0,0,0$ \\
\hline & - & {$[-1500: 1500]$} \\
\hline Observables & MSSM (GeV) & NHSSM $(\mathrm{GeV})$ \\
\hline$m_{\tilde{g}}$ & \multicolumn{2}{|c|}{2709} \\
\hline$m_{\tilde{t}_{1}}, m_{\tilde{t}_{2}}$ & $1120.2,2087.2$ & $1103.5,2087.2$ \\
\hline$m_{h}$ & 125.1 & 125.3 \\
\hline$m_{H}, m_{H^{ \pm}}$ & \multicolumn{2}{|c|}{1978,1979} \\
\hline $\mathrm{BR}\left(B \rightarrow X_{s}+\gamma\right)$ & $3.30 \times 10^{-4}$ & $3.50 \times 10^{-4}$ \\
\hline $\mathrm{BR}\left(B_{s} \rightarrow \mu^{+} \mu^{-}\right)$ & $3.20 \times 10^{-9}$ & $3.02 \times 10^{-9}$ \\
\hline
\end{tabular}

Table 1. Fixed input parameters used in this work (unless otherwise specified) for the MSSM and the NHSSM scenarios. Values for the masses and the trilinear coupling parameters are shown in $\mathrm{GeV}$. In both scenarios we set $\mu=200 \mathrm{GeV}(900 \mathrm{GeV}$ ) to ensure a higgsino (gaugino)-like neutralino LSP. Chosen values of $\tan \beta, M_{1}$ and $M_{2}$ are indicated in the text in individual contexts. SARAH (v4.10.2)-generated SPheno (v4.0.3) has been used to generate the particle spectra and for the estimation of the branching ratios and other observables. Parameter points are checked to be consistent with the current bounds as implemented in the packages HiggsBounds (v4.3.1) and HiggsSignals (v1.4.0).

\subsection{Pair-productions of sbottoms at the LHC}

In this section we study the effect of the NHSSM-specific parameters like $\mu^{\prime}$ and $A_{b}^{\prime}$ on the (parton-level) signal strengths $\left(\sim \sigma \times \mathrm{BR}^{2}\right)$ of sbottoms produced in pairs at the LHC and each decaying to a bottom quark and an LSP. This leads to a final state with $2 b$ jets + missing transverse energy $\left(\mathbb{E}_{T}\right)$ and is being intensively searched for at the LHC experiments leading to the strongest lower bound on the sbottom mass. Hence, as discussed in the Introduction, this might prove to be an appropriate process to find imprints of a scenario like the NHSSM. These could be in the form of yield in the above-mentioned final state that is different from the MSSM expectation, for a given set of masses of sbottoms, the lighter chargino and/or the lighter neutralinos. We, thus, study the relative yields in these 
two SUSY scenarios. This we do in stages. In section 3.3 .1 we stick to $\tilde{b}_{1}$ pair-production and consider cases with vanishing and non-zero $\mu^{\prime}$. $\tilde{b}_{2}$ pair-production is additionally considered in section 3.3.2 (for the simpler case with vanishing $\mu^{\prime}$ ) to demonstrate the situation when it cannot be ignored.

\subsubsection{The case with the lighter sbottom}

It may be reiterated that, at the lowest order in the perturbation theory, the NHSSM parameter $\mu^{\prime}$ affects only the electroweakino sector while the other NHSSM parameter in context, $A_{b}^{\prime}$, does so only for the sbottom sector of the said scenario. In contrast, ' $\mu$ ' affects both the sectors and does so in both MSSM and NHSSM. Furthermore, as we have already discussed in section $2, \tan \beta$ could also play an important role. Clearly, letting all the relevant parameters vary simultaneously and still be able to extract some concrete information is a difficult proposition. We, thus, need an appropriate strategy to obtain information which, if not free from, has less of an ambiguity and thus could be used to decipher an imprint of the NHSSM scenario in the experimental data. Note that we would, all through (unless otherwise specified), assume $m_{\tilde{b}_{L}}=m_{\tilde{b}_{R}}=1.2 \mathrm{TeV}$ which approximately sets the magnitude of $m_{\tilde{b}_{1}}$ to be around $1 \mathrm{TeV}$ (thus, evading the current bounds from the LHC) even in presence of maximal mixing between the two chiral states. All through we consider a vanishing $A_{b}$ (unless otherwise mentioned) given that it is found to play only a subdominant role in the sbottom sector.

First, for simplicity, we consider $\mu^{\prime}=0$. This is what we have already adopted in sections 3.1 and 3.2. Furthermore, to stick to a 'natural' setup we restrict ourselves to relatively small values of ' $\mu$ ' $(\lesssim 350 \mathrm{GeV})$. The soft gaugino parameters $M_{1}$ and $M_{2}$ are fixed at $500 \mathrm{GeV}$ and $1.1 \mathrm{TeV}$, respectively, thus rendering the two lighter neutralinos and the lighter chargino higgsino-like. ${ }^{6}$

In figure 9 we present a comparative study of the parton-level yields $\left(\sigma_{\tilde{b}_{1} \tilde{b}_{1}} \times \operatorname{BR}\left[\tilde{b}_{1} \rightarrow\right.\right.$ $\left.b \chi_{1}^{0}\right]^{2}$ ) in the final state $2 b+\mathbb{E}_{T}$ arising from pair-produced $\tilde{b}_{1}$ at the $13 \mathrm{TeV}$ run of the LHC in the MSSM (left) and the NHSSM (right) for $\tan \beta=10$ (top) and 40 (bottom). As mentioned earlier, the spectra, the branching ratios of various new-physics excitations and values of other low-energy observables are computed using SARAH-generated SPheno and the cross sections for the relevant processes are calculated using MadGraph5_aMC@NLO (v2.6.0) [81,82] with its default setup. Magnitudes of the yield are indicated via the color palettes in the plane of the input parameter ' $\mu$ ' $\left(\mu+A_{b}^{\prime}\right.$, for the plots on right) and the resulting mass-split between $\tilde{b}_{1}$ and the LSP, $\Delta m_{\left(\tilde{b}_{1}, \chi_{1}^{0}\right)}$. For the purpose, we varied ' $\mu$ ' and $A_{b}^{\prime}$ over the following ranges: $100 \mathrm{GeV} \leq \mu \leq 350 \mathrm{GeV}$ and $\left|A_{b}^{\prime}\right| \leq 1.2 \mathrm{TeV}$. The former would ensure the scenario to be 'natural'. The range of $A_{b}^{\prime}$ ensures no appearance of tachyonic sbottom states or CCB minima of the scalar potential while causing appropriate scalar mixing that are instrumental to the present analysis.

\footnotetext{
${ }^{6}$ In practice, one could have also considered a scenario with $M_{1}, M_{2} \ll \mu(\lesssim 500 \mathrm{GeV})$ thus making the light electroweakinos gaugino-like but still the overall scenario remaining somewhat "natural". However, in that case, one would not be able to witness the key effect of an enhanced $y_{b}$ in the NHSSM scenario as the decay of the bottom squarks would be mostly driven by appropriate gauge couplings.
} 

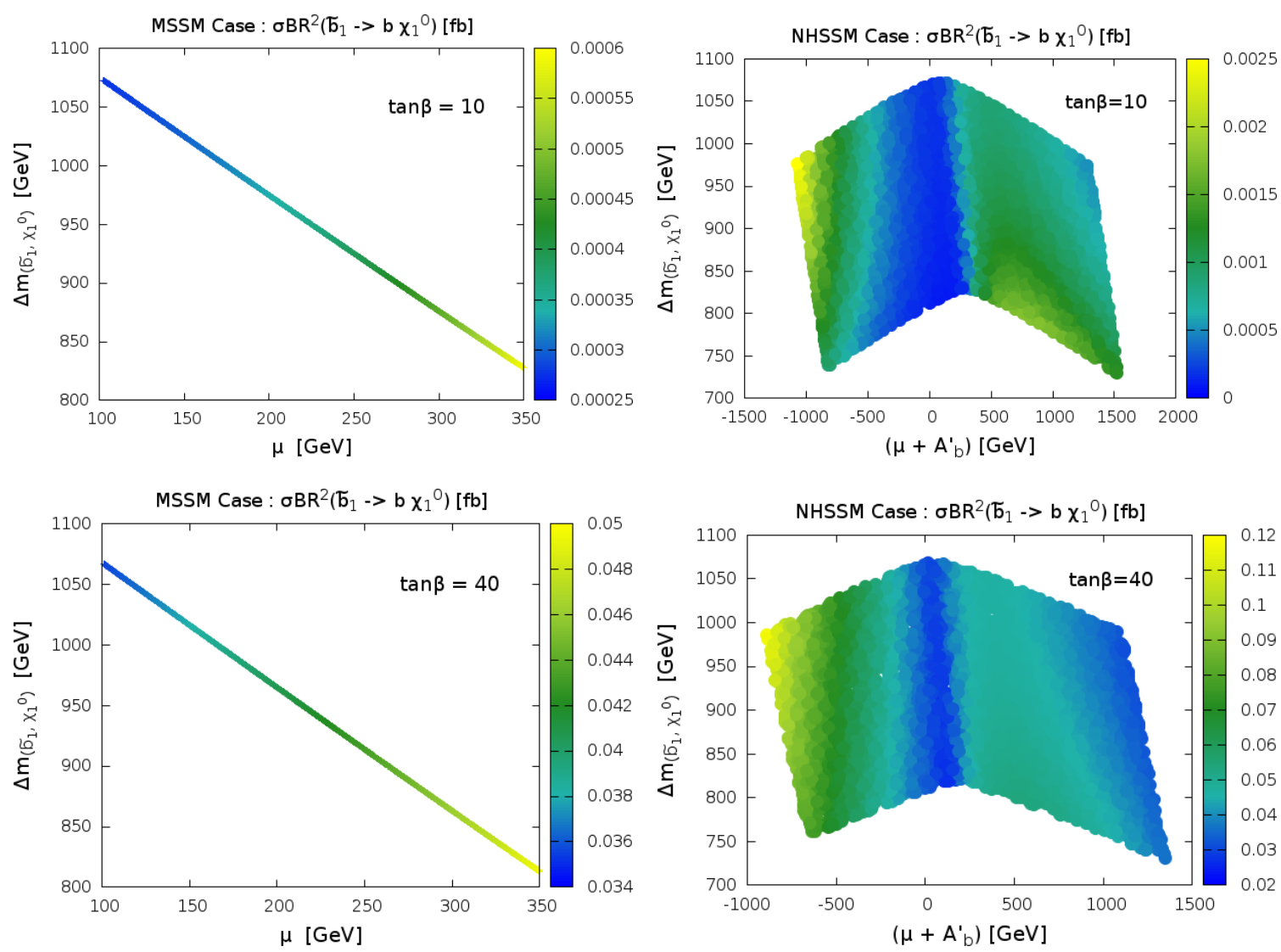

Figure 9. Parton-level yields $\left(\sigma_{\tilde{b}_{1} \tilde{b}_{1}} \times \mathrm{BR}\left[\tilde{b}_{1} \rightarrow b \chi_{1}^{0}\right]^{2}\right)$ in the final state $2 b+\mathbb{E}_{T}$ arising from pairproduced $\tilde{b}_{1}$ at the $13 \mathrm{TeV}$ run of the LHC in the MSSM (left) and the NHSSM (right) for $\tan \beta=10$ (top panel) and 40 (bottom panel). Magnitudes of the yield are indicated via the color palettes in the plane of the input parameter ' $\mu$ ' $\left(\mu+A_{b}^{\prime}\right.$, for the plots on right) and the resulting mass-split between $\tilde{b}_{1}$ and the LSP, $\Delta m_{\left(\tilde{b}_{1}, \chi_{1}^{0}\right)}$. Other fixed parameters are: $\mu=200 \mathrm{GeV}, M_{1}=500 \mathrm{GeV}$, $M_{2}=1.1 \mathrm{TeV}, A_{t}=2 \mathrm{TeV}, A_{b}=0, m_{\tilde{b}_{L}}=m_{\tilde{b}_{R}}=1.2 \mathrm{TeV}$ and $\mu^{\prime}=0$.

By comparing the left and the right plots in a given row (i.e., for a given $\tan \beta$ ) of figure 9, it is clear that the yield in the NHSSM for a particular value of ' $\mu$ ' (i.e., $m_{\chi_{1}^{0}} \simeq m_{\chi_{1}^{ \pm}}$, for $\mu \ll M_{1}, M_{2}$ ) could vary widely as opposed to a rather definite expectation for the same in the MSSM. This becomes clear from the plots on the right in which the horizontal axes depict the variation of the NHSSM construct $\mu+A_{b}^{\prime}$ that appears in the off-diagonal term of the symmetric sbottom mass-squared matrix and augments the corresponding MSSM matrix for which only ' $\mu$ ' appears. The vertical bands in blue in the central region of the plots on the right with smaller values of $\mu+A_{b}^{\prime}$ are indicative of the smallest yields. These are commensurate with a small mixing in the sbottom sector over this region. As discussed in section 3.1, such a small mixing leaves $\tilde{b}_{1}$ dominantly left-handed and hence with an enhanced $\operatorname{BR}\left[\tilde{b}_{1} \rightarrow t \chi_{1}^{-}\right]$. Consequently, one finds a suppressed $\operatorname{BR}\left[\tilde{b}_{1} \rightarrow b \chi_{1}^{0}\right]$ over this region. We have checked that the patterns shown in the plots on the right closely follow the same for the above branching fraction. The latter, in the first place, inherits its pattern 
from those of the interaction strengths as illustrated in figure 3. Such a similarity is seen for large negative values of $\mu+A_{b}^{\prime}$ (i.e., for large negative $A_{b}^{\prime}$ ) when we obtain the highest yield (in yellow).

As for the MSSM case (i.e., the plots on the left), it may further be noted that the variations in $\sigma_{\tilde{b}_{1} \tilde{b}_{1}} \times \mathrm{BR}\left[\tilde{b}_{1} \rightarrow b \chi_{1}^{0}\right]^{2}$, i.e., the parton-level yields, are primarily due to variations in $\mathrm{BR}\left[\tilde{b}_{1} \rightarrow b \chi_{1}^{0}\right]$ which, in turn, are attributed to the changing chiral contents of $\tilde{b}_{1}$ as ' $\mu$ ' varies. This is clear since the available phase space $\left(\Delta m_{\left(\tilde{b}_{1}, \chi_{1}^{0}\right)}\right)$ for the above decay remains to be large enough over the entire range of variation of ' $\mu$ '. This also lends credence to the fact that the (counter-intuitive) increase in the yield at lower values of $\Delta m_{\left(\tilde{b}_{1}, \chi_{\tilde{\sigma}}^{0}\right)}$ (i.e., decreasing phase space for the decay) is actually connected to the composition of $\tilde{b}_{1}$ in these regions, as discussed above. In summary, for a given set of values of $m_{\tilde{b}_{1}}$ and $m_{\chi_{1}^{0}}$ where the latter is higgsino-like and relatively light, the yields in the NHSSM could surpass their corresponding MSSM expectations by large margins. However, the magnitudes of $\sigma \times \mathrm{BR}$ reveals that these are somewhat sensitive to the LHC experiments with $300 \mathrm{fb}^{-1}$ of integrated luminosity only when $\tan \beta$ is reasonably large.

Next, we bring in $\mu^{\prime}$ into the picture. As pointed out in section 2 , this could give rise to a relatively heavier higgsino-like neutralino $(\sim 1 \mathrm{TeV})$ LSP without requiring ' $\mu$ ' to be large $[43,46]$. This would then help avoid an imminent tension with the notion of 'naturalness'. In addition, such a neutralino LSP is known to be a viable DM candidate that could explain the observed relic density. In view of the LHC data routinely pushing up the lower bounds on sparticle masses and that a bino-dominated neutralino LSP overproduces the relic abundance, these together certainly offer an welcome respite. Furthermore, a non-vanishing $\mu^{\prime}$ would not by itself affect the electroweakino sector in the sense that the latter is blind to ' $\mu$ ' or $\mu^{\prime}$ separately but inherits its properties only from the sum $\mu+\mu^{\prime}$. However, of ' $\mu$ ' and $\mu^{\prime}$, only the former enters the sbottom sector, at least at the lowest order. Such a selectiveness is expected to leave its trails in the masses and interactions involving these two sectors and the way they are connected. These would then carry imprints of the NHSSM scenario and could even shed light on the relative magnitude of ' $\mu$ ' and $\mu$ '.

In figure 10 we present the possible extent of variation of the yield $\left(\sigma \times \mathrm{BR}^{2}\right)$ as a function of $\mu^{\prime}$ and $A_{b}^{\prime}$ for $m_{\tilde{b}_{L}}=m_{\tilde{b}_{R}}=1.2 \mathrm{TeV}$. This is done by fixing the MSSM parameters ' $\mu$ ', $M_{1}$ and $A_{b}$ in the following way: $\mu=200 \mathrm{GeV}, M_{1}=500 \mathrm{GeV}$ and $A_{b}=0$. All other input parameters are as indicated in table 1 . Note that the chosen (small) value of ' $\mu$ ' is expected to render the scenario 'natural'. All these, in turn, fix the MSSM yield. The plot on the left (right) corresponds to $\tan \beta=10$ (40). By fixing $M_{1}$ and allowing for $\mu^{\prime}$ to vary about it, we essentially let the nature of the LSP neutralino change over from a dominantly higgsino-like one to a gaugino-like one passing through an intermediate state of mixed nature and thus, capture how the yield varies with such a change. Plots in the bottom panel zoom in on the higgsino-like LSP region. The white bands in the middle of the plots indicate regions which are excluded by the LEP chargino searches [83]. Fixed $m_{\tilde{b}_{1}}$ contours are also overlaid in each plot. 

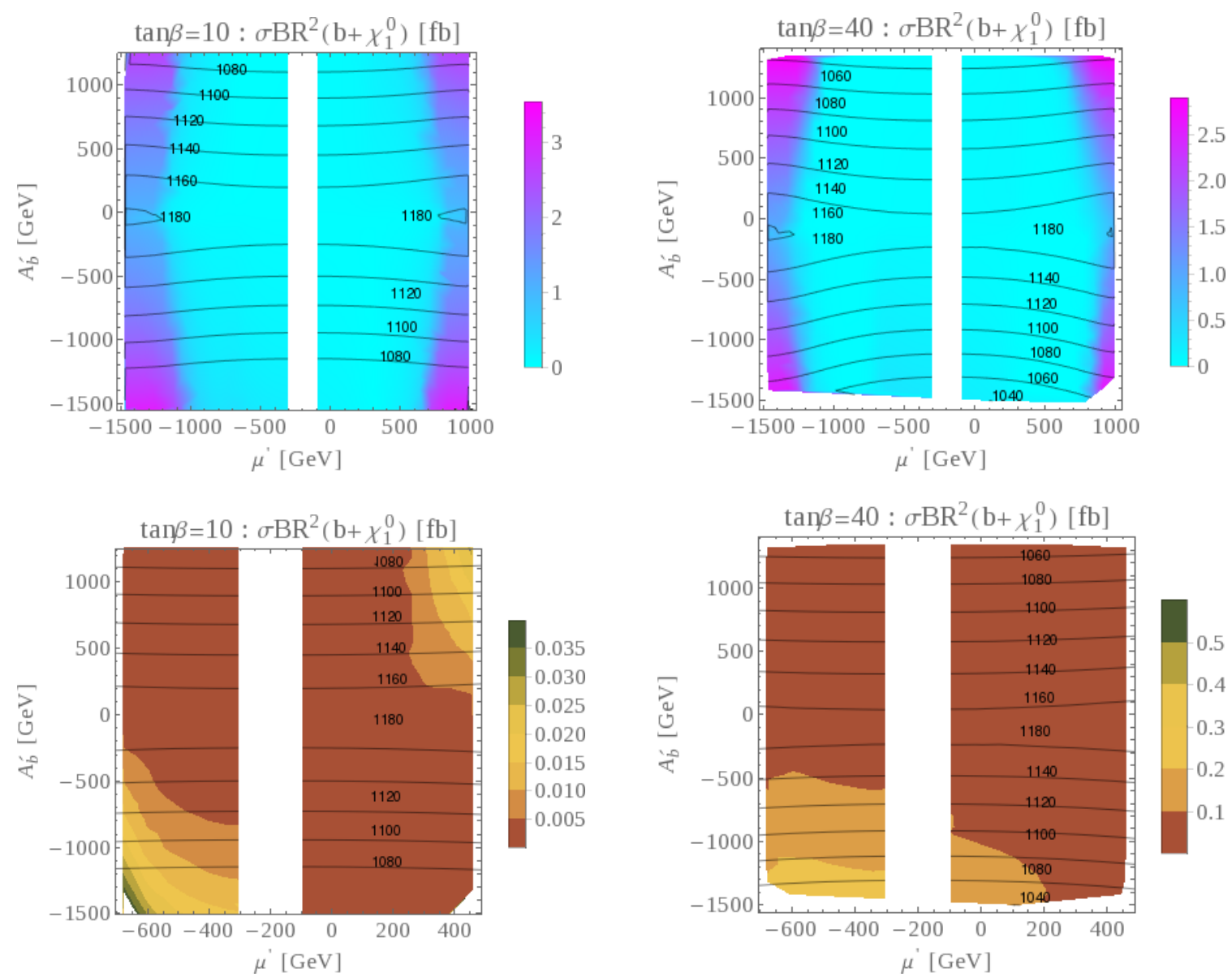

Figure 10. Variation of $\sigma_{\tilde{b}_{1} \tilde{b}_{1}} \times \mathrm{BR}\left[\tilde{b}_{1} \rightarrow b \chi_{1}^{0}\right]^{2}$ (yield) in the $2 b+\mathscr{E}_{T}$ final state as a function of $\mu^{\prime}$ and $A_{b}^{\prime}$ for fixed MSSM configurations $\left(\mu=200 \mathrm{GeV}, M_{1}=500 \mathrm{GeV}, m_{\tilde{b}_{L}}=m_{\tilde{b}_{R}}=1.2 \mathrm{TeV}, A_{b}=0\right.$ with $\tan \beta=10$ (left) and 40 (right)) over an extended range of $\mu^{\prime}$ (top panel) and for a zoomed-up range with low $\mu^{\prime}$ (bottom panel). The blank vertical bands in the middle are roughly excluded by searches of the lighter chargino at the LEP experiments, i.e., $m_{\chi_{1}^{ \pm}} \gtrsim 100 \mathrm{GeV}$ [83]. See table 1 and the text for details.

In both plots of the top panel of figure 10, the regions in cyan are of the smallest yields. The fixed MSSM yields for both $\tan \beta=10$ and 40 are obtained for $\mu^{\prime}=A_{b}^{\prime}=0$ and those are as small as $0.0003 \mathrm{fb}$ and $0.039 \mathrm{fb}$, respectively. We have checked that the smallness of the yield over the cyan region is mostly due to the same for the effective interaction strength $C_{L}^{2}+C_{R}^{2}$. Even the presence of a slightly darker vertical shade in the middle of the plot on the right indicating locally enhanced yields over the range $-700 \mathrm{GeV} \lesssim \mu^{\prime} \lesssim 400 \mathrm{GeV}$ and for $A_{b}^{\prime}<0$ can be traced back to similar enhancements in $C_{L}^{2}+C_{R}^{2}$. Also, note that the magnitude of the yield varies in a discontinuous fashion with increasing $\left|\mu^{\prime}\right|$. This is due to an interplay between the $y_{b}$-driven contribution which falls off more rapidly with growing $\left|\mu^{\prime}\right|$ (because of a decreasing higgsino content of the LSP) than what the growing gaugino (bino) contribution could compensate for. Note that even though the gaugino-dominance sets in at length for larger values of $\left|\mu^{\prime}\right|$ and takes control over the yield, 
its 'extreme' values draw heavily (though indirectly) from the ever-diminishing branching fraction $\operatorname{BR}\left[\tilde{b}_{1} \rightarrow t \chi_{1}^{-}\right]$as $m_{\chi_{1}^{ \pm}}$grows with $\left|\mu^{\prime}\right|$, before getting kinematically forbidden. In the bottom panel of figure 10 we zoom up the low $\left|\mu^{\prime}\right|$ region to illustrate the altering nature of the yield and its extent across the region. It can be gleaned from these plots that a 5 to 7 fold change in the yield is possible over the indicated range.

In summary, an enhanced $y_{b}$, which is rather characteristic of the NHSSM scenario for large negative $A_{b}^{\prime}$ and large $\tan \beta$, could boost the yield in the $2 b+\mathbb{E}_{T}$ final state beyond its MSSM expectation, for similar masses of the lighter sbottom and the LSP in the two scenarios. The reverse is true when $A_{b}^{\prime}$ takes large positive values. In particular, the enhancement is significant for a higgsino-like LSP and is somewhat more interesting when the lighter sbottom is $\tilde{b}_{L}$-dominated given that there is a competition among the decays $\tilde{b}_{1} \rightarrow b \chi_{1,2}^{0}$ and $\tilde{b}_{1} \rightarrow t \chi_{1}^{-}$.

\subsubsection{Impact of including the heavier sbottom}

A priori, it would not be fair to ignore the contribution from $p p \rightarrow \tilde{b}_{2} \tilde{b}_{2}^{*}$. This is since, for the ranges of variations of various parameters (like $A_{b}^{\prime}$ and $\tan \beta$ ), $m_{\tilde{b}_{1}}$ and $m_{\tilde{b}_{2}}$ may not be too different, in particular, when we consider $m_{\tilde{b}_{L}}$ and $m_{\tilde{b}_{R}}$ to be degenerate. It would thus be instructive to check what role could $\tilde{b}_{2}$ possibly play in the phenomenology.

To illustrate this, in the left plot of figure 11 we present the contours of constant mass-split $\left(\Delta m_{\left(\tilde{b}_{2}, \tilde{b}_{1}\right)}\right)$ between $\tilde{b}_{2}$ and $\tilde{b}_{1}$ in the $A_{b}^{\prime}-\tan \beta$ plane. We note that for the extreme value for $\left|A_{b}^{\prime}\right|(=1.2 \mathrm{TeV})$ that we have allowed for in the present analysis, the split between $m_{\tilde{b}_{1}}$ and $m_{\tilde{b}_{2}}$ cannot be more than around $170 \mathrm{GeV}$. Furthermore, as can be seen in figure 11, the mass-split is largely independent of $\tan \beta$. Thus, for low values of $\left|A_{b}^{\prime}\right|, m_{\tilde{b}_{1}}$ and $m_{\tilde{b}_{2}}$ could lie very close by and hence $\sigma\left(p p \rightarrow \tilde{b}_{2} \tilde{b}_{2}^{*}\right)$ may turn out to be not much smaller than $\sigma\left(p p \rightarrow \tilde{b}_{1} \tilde{b}_{1}^{*}\right)$. These cross sections, as functions of $A_{b}^{\prime}$, are compared in the right plot of figure 11 for a fixed value of $\tan \beta=40$. Bands arise due to variation of ' $\mu$ ' over the range $100 \mathrm{GeV} \leq \mu \leq 350 \mathrm{GeV}$. We have checked that the pattern of variation or the values of cross sections do not alter much if a different value of $\tan \beta$ is chosen. This is not unexpected since the production cross sections are mainly governed by the masses of the sbottoms. What then could matter for the yield in the final state of our interest, i.e., $2 b+\mathbb{E}_{T}$, is $\operatorname{BR}\left[\tilde{b}_{2} \rightarrow b \chi_{1}^{0}\right]$.

In figure 12 we present the variations of branching fractions $\operatorname{BR}\left[\tilde{b}_{1} \rightarrow b \chi_{1}^{0}\right]$ and $\operatorname{BR}\left[\tilde{b}_{2} \rightarrow\right.$ $\left.b \chi_{1}^{0}\right]$ as functions of $A_{b}^{\prime}$ for $\tan \beta=10$ and 40 . Unlike the variations of their pair-production cross sections, these show some significant quantitative difference between the cases with $\tan \beta=10$ and 40 . As can be seen, $\operatorname{BR}\left[\tilde{b}_{2} \rightarrow b \chi_{1}^{0}\right]$ is always larger than $\operatorname{BR}\left[\tilde{b}_{1} \rightarrow b \chi_{1}^{0}\right]$ for $A_{b}^{\prime}<0$. The largest difference is seen around vanishing $A_{b}^{\prime}$ where $\operatorname{BR}\left[\tilde{b}_{2} \rightarrow b \chi_{1}^{0}\right]$ peaks while $\mathrm{BR}\left[\tilde{b}_{2} \rightarrow b \chi_{1}^{0}\right]$ touches the minimum. The phenomenon could be understood in terms of the sharply increasing dominance of $\tilde{b}_{R}$ in $\tilde{b}_{2}$ as $\left|A_{b}^{\prime}\right| \rightsquigarrow 0$. This quickly suppresses $\operatorname{BR}\left[\tilde{b}_{2} \rightarrow t \chi_{1}^{-}\right]$in favor of $\operatorname{BR}\left[\tilde{b}_{2} \rightarrow b \chi_{1}^{0}\right]$. The situation is just the opposite in the case of $\operatorname{BR}\left[\tilde{b}_{1} \rightarrow b \chi_{1}^{0}\right]$ thus leading to the contrast seen for the two variations. Such an enhanced magnitude of $\mathrm{BR}\left[\tilde{b}_{2} \rightarrow b \chi_{1}^{0}\right]$ for $A_{b}^{\prime}<0$ could very well compensate for a relatively smaller value of $\sigma\left(p p \rightarrow \tilde{b}_{2} \tilde{b}_{2}^{*}\right)$. Thus, the yield in the $2 b+\mathbb{E}_{T}$ final state from $\tilde{b}_{1} \tilde{b}_{1}^{*}$ and $\tilde{b}_{2} \tilde{b}_{2}^{*}$ could be comparable and hence the latter should not be ignored. 

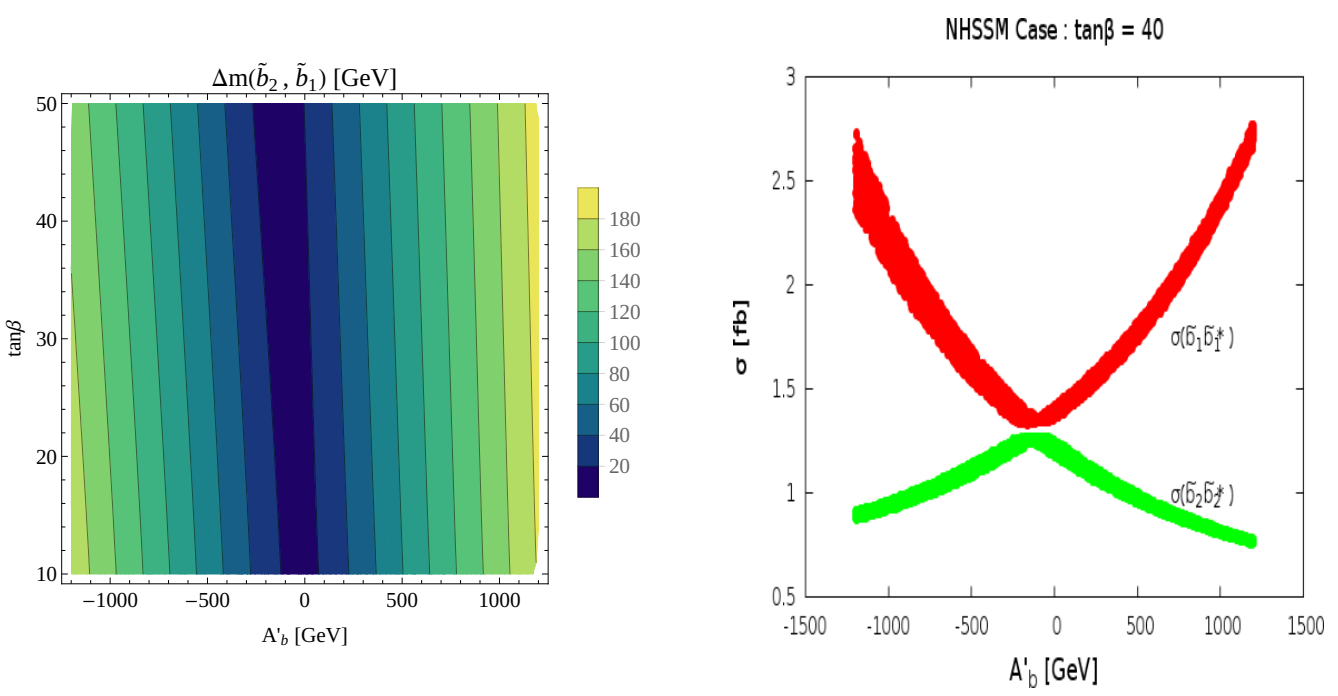

Figure 11. Contours of constant $\Delta m_{\left(\tilde{b}_{2}, \tilde{b}_{1}\right)}$ in the $A_{b}^{\prime}-\tan \beta$ plane (left) and variations of $\sigma(p p \rightarrow$ $\left.\tilde{b}_{1} \tilde{b}_{1}^{*}, \tilde{b}_{2} \tilde{b}_{2}^{*}\right)$ as functions of $A_{b}^{\prime}$ for $\tan \beta=40$ and $100 \mathrm{GeV} \leq \mu \leq 350 \mathrm{GeV}$. For both plots $m_{\tilde{b}_{L}}=$ $m_{\tilde{b}_{R}}=1.2 \mathrm{TeV}$. Other fixed parameters are $A_{b}=0, \mu=200 \mathrm{GeV}, \mu^{\prime}=0, A_{t}=2 \mathrm{TeV}, M_{1}=500 \mathrm{GeV}$ and $M_{2}=1.1 \mathrm{TeV}$.
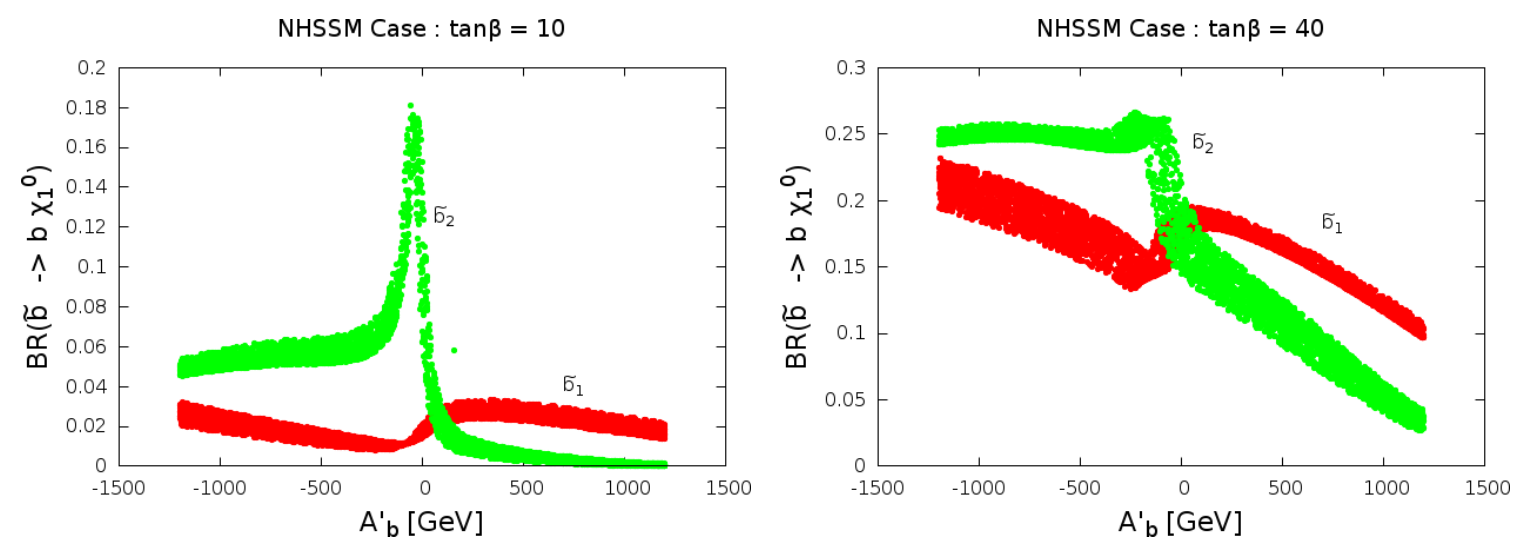

Figure 12. Branching fractions of $\tilde{b}_{1}\left(\tilde{b}_{2}\right)$ to $b \chi_{1}^{0}$ shown in red (green) as functions of $A_{b}^{\prime}(\mathrm{GeV})$. The left (right) panel stands for $\tan \beta=10$ (40). Other fixed parameters are: $M_{1}=500 \mathrm{GeV}$, $M_{2}=1.1 \mathrm{TeV}, A_{t}=2 \mathrm{TeV}, A_{b}=0, m_{\tilde{b}_{L}}=m_{\tilde{b}_{R}}=1.2 \mathrm{TeV}$ and $\mu^{\prime}=0$ with $100 \leq \mu \leq 350 \mathrm{GeV}$.

In figure 13 we show the variations of the parton-level yields (i.e., the cross section times the square of $\mathrm{BR}\left[\tilde{b}_{1 / 2} \rightarrow b \chi_{1}^{0}\right]$ ) in the $2 b+\mathbb{E}_{T}$ final state arising from $\tilde{b}_{1^{-}}$(in red) and $\tilde{b}_{2}$ (in green) pair-productions for $\tan \beta=10$ (40) in the left (right) plot. For the plot on the right, we also added up these two contributions (in blue) as in this case the individual contributions are relatively large over the range we explored. As one can see, for small values of $\left|A_{b}^{\prime}\right|$, yield from $\tilde{b}_{2}$ pair-production dominates and this simply inherits its trend from the plot on the right of figure 11 and from figure 12. However, with small $\left|A_{b}^{\prime}\right|$ the scenario tends to become MSSM-like over this region. Interestingly, for relatively large negative $A_{b}^{\prime}$ and for large $\tan \beta$ the combined contribution from $\tilde{b}_{1}$ and $\tilde{b}_{2}$ pair-production 

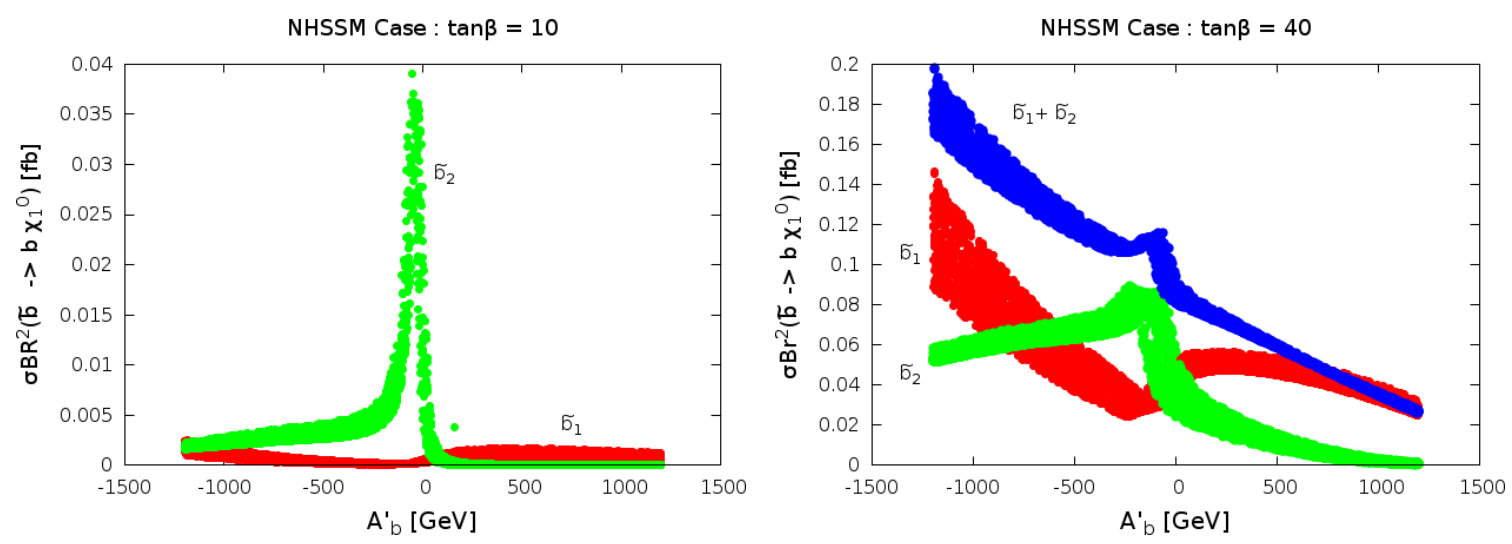

Figure 13. Variation of parton-level yields in the $2 b+\chi_{1}^{0}$ final state arising from $\tilde{b}_{1}$ (in red) and $\tilde{b}_{2}$ (in green) pair-production at the $13 \mathrm{TeV}$ LHC as functions of $A_{b}^{\prime}$. The left (right) panel stands for $\tan \beta=10(40)$. Other fixed/varying parameters are as in figure 12. For the plot on the right, contributions from $\tilde{b}_{1}$ and $\tilde{b}_{2}$ are added up (in blue).
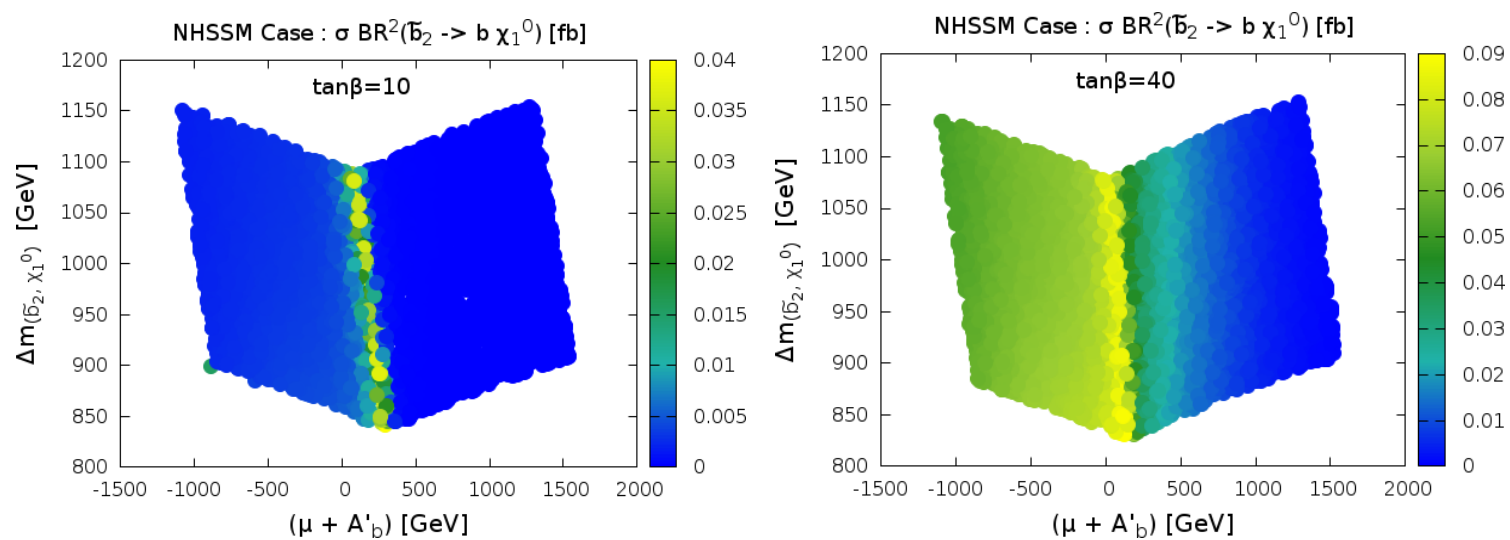

Figure 14. Parton-level yields $\left(\sigma_{\tilde{b}_{2} \tilde{b}_{2}^{*}} \times \mathrm{BR}\left[\tilde{b}_{2} \rightarrow b \chi_{1}^{0}\right]^{2}\right)$ in the final state $2 b+\mathbb{E}_{T}$ arising from pair-produced $\tilde{b}_{2}$ at the $13 \mathrm{TeV}$ run of the LHC in the NHSSM for $\tan \beta=10$ (left panel) and 40 (right panel) in the plane of $\mu+A_{b}^{\prime}$ and the resulting mass-split between $\tilde{b}_{2}$ and the LSP, $\Delta m_{\left(\tilde{b}_{2}, \chi_{1}^{0}\right)}$. Magnitudes of the yield are indicated via color palettes. Other fixed/varying parameters are as in figure 12 .

could exceed the MSSM expectation significantly. For a given set of values of $m_{\tilde{b}_{1}}, m_{\tilde{b}_{2}}$ and $m_{\chi_{1}^{0}}$, this then could signal in favour of a scenario like the NHSSM with a few tens of events in $300 \mathrm{fb}^{-1}$ of data at the LHC. On the other hand, for large positive $A_{b}^{\prime}$, one expects a significant dearth of events in the $2 b+\mathbb{E}_{T}$ final state when compared to the MSSM expectation. However, the yield is found to be not sensitive to $300 \mathrm{fb}^{-1}$ of data and would need the high luminosity run of the LHC for this to show up. In figure 14 we illustrate the variation of $\sigma_{\tilde{b}_{2} \tilde{b}_{2}^{*}} \times \operatorname{BR}\left[\tilde{b}_{2} \rightarrow b \chi_{1}^{0}\right]^{2}$ in the $\left(\mu+A_{b}^{\prime}\right)-\Delta m_{\left(\tilde{b}_{2}, \chi_{1}^{0}\right)}$ plane for $\tan \beta=10$ (left) and 40 (right). These correspond to the similar ones for $\tilde{b}_{1}$ presented in the right plots in the top and bottom panels of figure 9 .

We now summarize our findings by undertaking a simple-minded comparison of the $2 b+\mathbb{E}_{T}$ rates obtained in the MSSM and in the NHSSM, as $A_{b}^{\prime}$ varies for the same values 
of $\mu$ (i.e., similar higgsino-like LSP masses). We define the relative rates as

$$
\begin{aligned}
\alpha_{i}\left(A_{b}^{\prime}\right)= & \frac{\left[\left(\sigma_{\tilde{b}_{i} \tilde{b}_{i}} \times \mathrm{BR}\left[\tilde{b}_{i} \rightarrow b \tilde{\chi}_{1}^{0}\right]^{2}\right)\right]^{\mathrm{NHSSM}}}{\left[\left(\sigma_{\tilde{b}_{i} \tilde{b}_{i}} \times \mathrm{BR}\left[\tilde{b}_{i} \rightarrow b \tilde{\chi}_{1}^{0}\right]^{2}\right)\right]^{\mathrm{MSSM}}} \text { and } \\
\alpha_{\text {total }}\left(A_{b}^{\prime}\right)= & \frac{\sum_{i=1,2}\left[\left(\sigma_{\tilde{b}_{i} \tilde{b}_{i}} \times \mathrm{BR}\left[\tilde{b}_{i} \rightarrow b \tilde{\chi}_{1}^{0}\right]^{2}\right)\right]^{\mathrm{NHSSM}}}{\sum_{i=1,2}\left[\left(\sigma_{\tilde{b}_{i} \tilde{b}_{i}} \times \mathrm{BR}\left[\tilde{b}_{i} \rightarrow b \tilde{\chi}_{1}^{0}\right]^{2}\right)\right]^{\mathrm{MSSM}}}
\end{aligned}
$$

with $m_{\tilde{b}_{L}}=m_{\tilde{b}_{R}}=1.2 \mathrm{TeV}$ and its variation is shown in figure 15 in the plane of $\mu+A_{b}^{\prime}$ and $\Delta m_{\left(\tilde{b}_{1}, \chi_{1}^{0}\right)}$. We obtain the rates in the MSSM case by varying ' $\mu$ ' over the range $100 \mathrm{GeV} \leq \mu \leq 350 \mathrm{GeV}$ such that a relatively light higgsino-like neutralino LSP is ensured. For the NHSSM case, we choose $\mu^{\prime}=0$ but vary $A_{b}^{\prime}$ such that $\left|A_{b}^{\prime}\right| \leq 1.2 \mathrm{TeV}$ to get the yields. To compute ' $\alpha$ ', the ratios are taken of those rates in the two scenarios for which values of ' $\mu$ ' are the same. The plots reveal that up to a eight-fold (six-fold) increased rates could be possible for $\tan \beta=10$ (40) over the expected MSSM rates in the final state under consideration. These further indicate that NHSSM could also result in a much lower yield when compared to the MSSM. Note, however, that the absolute rates are typically lower for a smaller $\tan \beta$. Understandably, the largest deviation is expected for relatively large, negative values of $A_{b}^{\prime}$ for which $y_{b}$ is much enhanced. It should be noted that the variations of ' $\alpha$ ' closely mimics that of $\sigma \times \mathrm{BR}^{2}$ in figures 9 and 14 . Hence the former variations find very similar explanations in terms of variation of the effective interaction strengths as well.

Finally, it will be of practical importance to understand how the rates would compare when the masses of the sbottoms vary. To this end, in figure 16 we illustrate the variations of a similar ratio of the expected (total) yields as defined in equation (3.3) (but for two representative choices of $A_{b}^{\prime}$ and $\left.\tan \beta=40\right)$ in the plane of $m_{\tilde{b}_{L}}$ and $m_{\tilde{b}_{R}}$. Resulting contours of fixed $m_{\tilde{b}_{1}}$ and $m_{\tilde{b}_{2}}$ are overlaid. One finds that for the plot on left with $A_{b}^{\prime}=-1 \mathrm{TeV}$, the maximal deviation in the yields in the $2 b$-jets $+\mathbb{E}_{T}$ final state with respect to the MSSM yield occurs for $\tilde{b}_{1}$-dominated by $\tilde{b}_{L}$ (black region). The reverse is true for the plot on the right with $A_{b}^{\prime}=1 \mathrm{TeV}$. Both of these situations could be understood in terms of the way $y_{b}$ varies with $A_{b}^{\prime}$ and the added role $A_{b}^{\prime}$ plays in the mixing in the sbottom sector in the NHSSM, over and above the already existing MSSM effects.

To elaborate a bit more, for $A_{b}^{\prime}=-1 \mathrm{TeV}$ as is used in the left plot, we now know that $y_{b}$ is significantly larger than its MSSM value. As the decay $\tilde{b} \rightarrow b \chi_{1}^{0}$ is enhanced for larger $y_{b}$, we always find the relative yield to be larger than 1 . It turns out that the maximal deviation (black region) occurs when the MSSM rate (appearing in the denominator of the ratio) in the said channel becomes minimum which is the case when $\tilde{b}_{1} \approx \tilde{b}_{L}$. This is so since such a limit is more abruptly attained in the MSSM than in the NHSSM thanks to a nonvanishing $A_{b}^{\prime}$ for which $\tilde{b}_{1}$ could still have a larger $\tilde{b}_{R}$ admixture in the NHSSM case which facilitates the decay $\tilde{b}_{1} \rightarrow b \chi_{1}^{0}$. In moving to the plot on the right, the MSSM contribution in the denominator does not change. However, with $A_{b}^{\prime}=1 \mathrm{TeV}, y_{b}$ becomes much smaller than its MSSM value. Thus, in the region for which $\tilde{b}_{1}$ becomes dominated by $\tilde{b}_{L}$ (left of the diagonal), $\tilde{b}_{1} \rightarrow t \chi_{1}^{-}$prevails. This is the reason the ratio of the yields are now smaller over this region. The maximal ratio (black patch) is now obtained for $\tilde{b}_{1} \approx \tilde{b}_{R}$ and this appears 

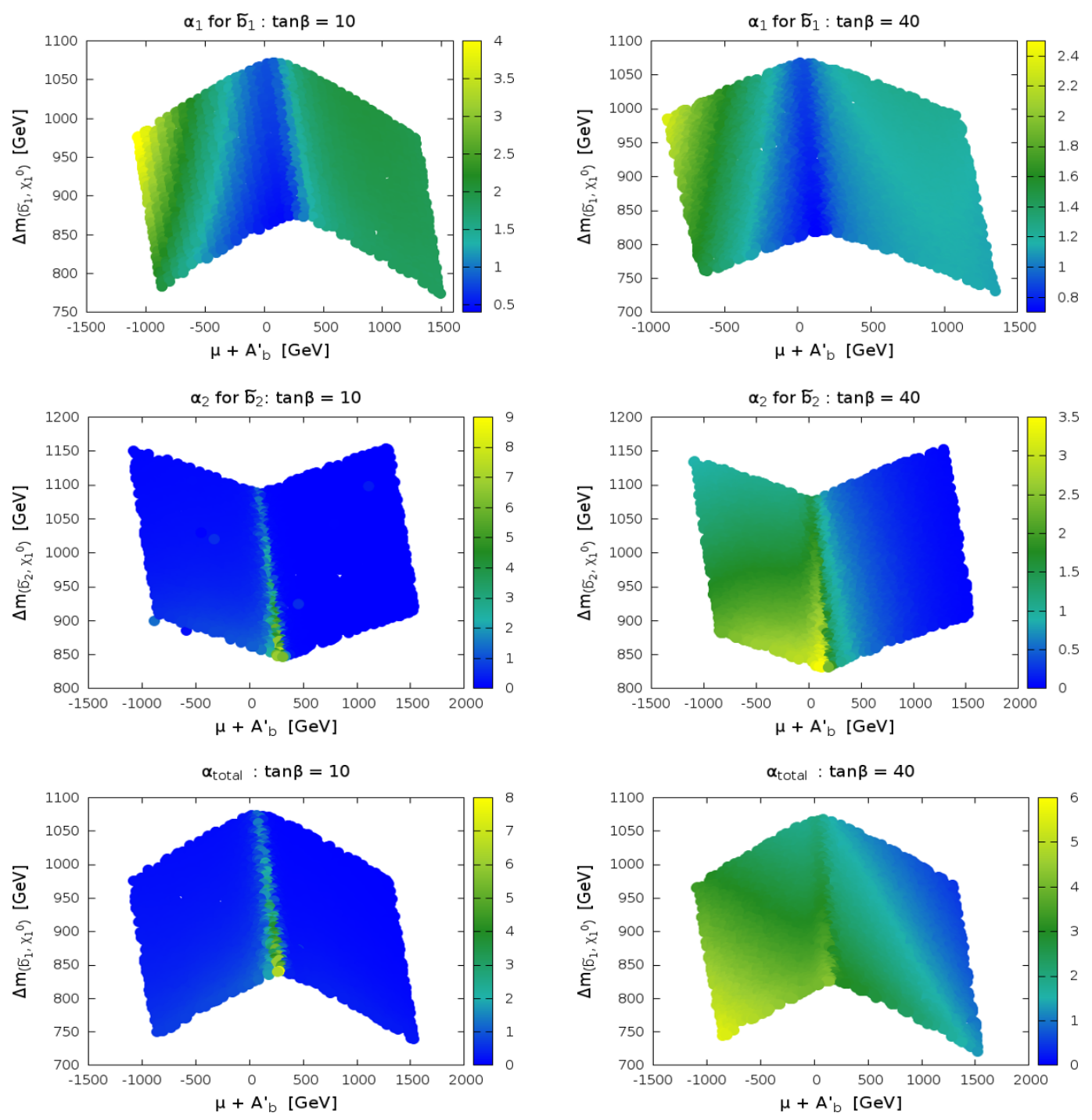

Figure 15. Relative rates $(\alpha)$ between the NHSSM and the MSSM scenarios in the final state $2 b+\mathbb{E}_{T}$ in the plane of $\mu+A_{b}^{\prime}$ and $\Delta m_{\left(\tilde{b}_{i}, \chi_{1}^{0}\right)}$ obtained from $\tilde{b}_{1}$ pair-production (top row), $\tilde{b}_{2}$ pairproduction (middle row) and sum of $\tilde{b}_{1}$ and $\tilde{b}_{2}$ pair-production (bottom row) for $\tan \beta=10$ (left panel) and 40 (right panel). ' $\mu$ ' is varied over the range $100 \mathrm{GeV} \leq \mu \leq 350 \mathrm{GeV}$ while the other fixed parameters are: $M_{1}=500 \mathrm{GeV}, M_{2}=1.1 \mathrm{TeV}, A_{t}=2 \mathrm{TeV}, A_{b}=0, m_{\tilde{b}_{L}}=m_{\tilde{b}_{R}}=1.2 \mathrm{TeV}$ and $\mu^{\prime}=0$.

entirely due to enhanced cross section in the NHSSM case where $\tilde{b}_{1}$ gets lighter than that in the case of the MSSM for the same input values of $m_{\tilde{b}_{L}}$ and $m_{\tilde{b}_{R}}$. Note that this effect is also there over the black patch in the left plot which gets further fortified by the effects discussed above. Clearly, these can turn the ratio bigger as can be seen for $A_{b}^{\prime}<0$.

\subsection{Implication for stop searches}

In this subsection we briefly discuss possible implications of the NHSSM scenario for the stop searches at the LHC. It has been pointed out in the Introduction that the impact of 

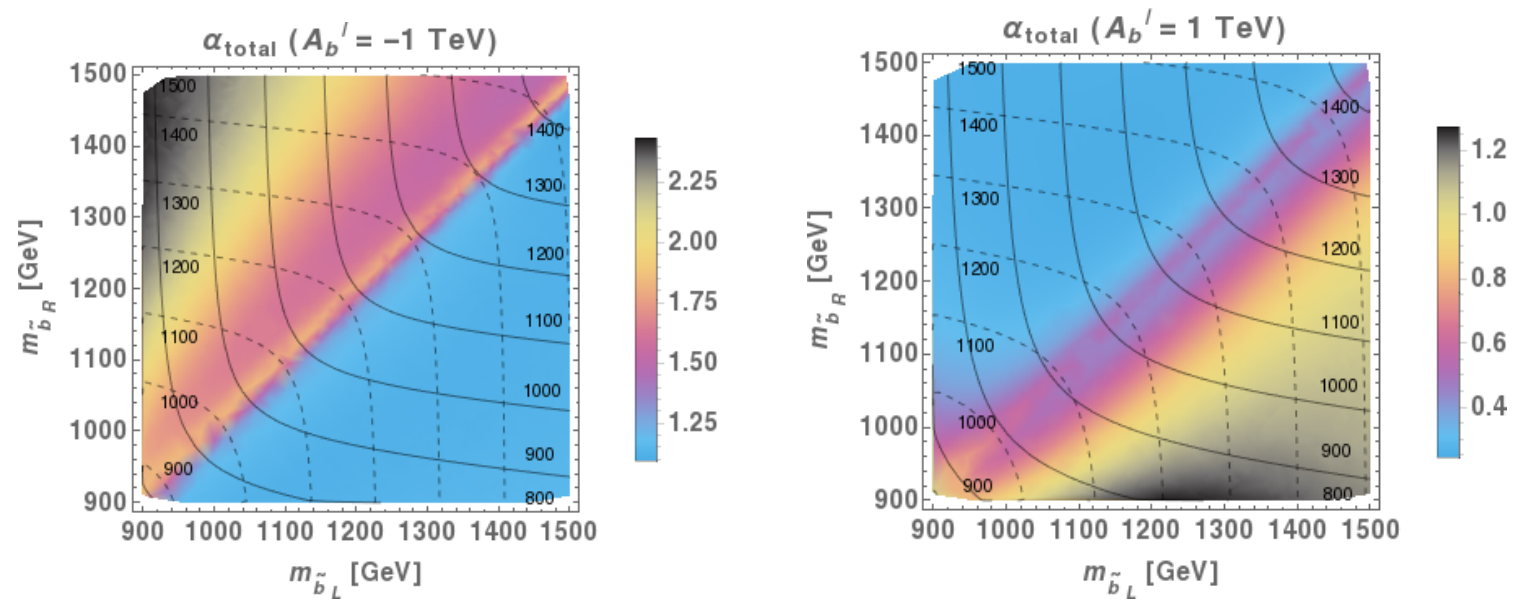

Figure 16. Variations of $\alpha_{\text {total }}$ in the NHSSM and in the MSSM in the $m_{\tilde{b}_{L}}-m_{\tilde{b}_{R}}$ plane for $A_{b}^{\prime}=-1 \mathrm{TeV}$ (left) and $A_{b}^{\prime}=1 \mathrm{TeV}$ (right) and for fixed values of $\tan \beta(=40)$ and $\mu(=200 \mathrm{GeV})$. Other fixed parameters are as in figure 15. Contours of constant $m_{\tilde{b}_{1}}\left(m_{\tilde{b}_{2}}\right)$ are overlaid with solid (dashed) lines along the right (left) edges of the plots.
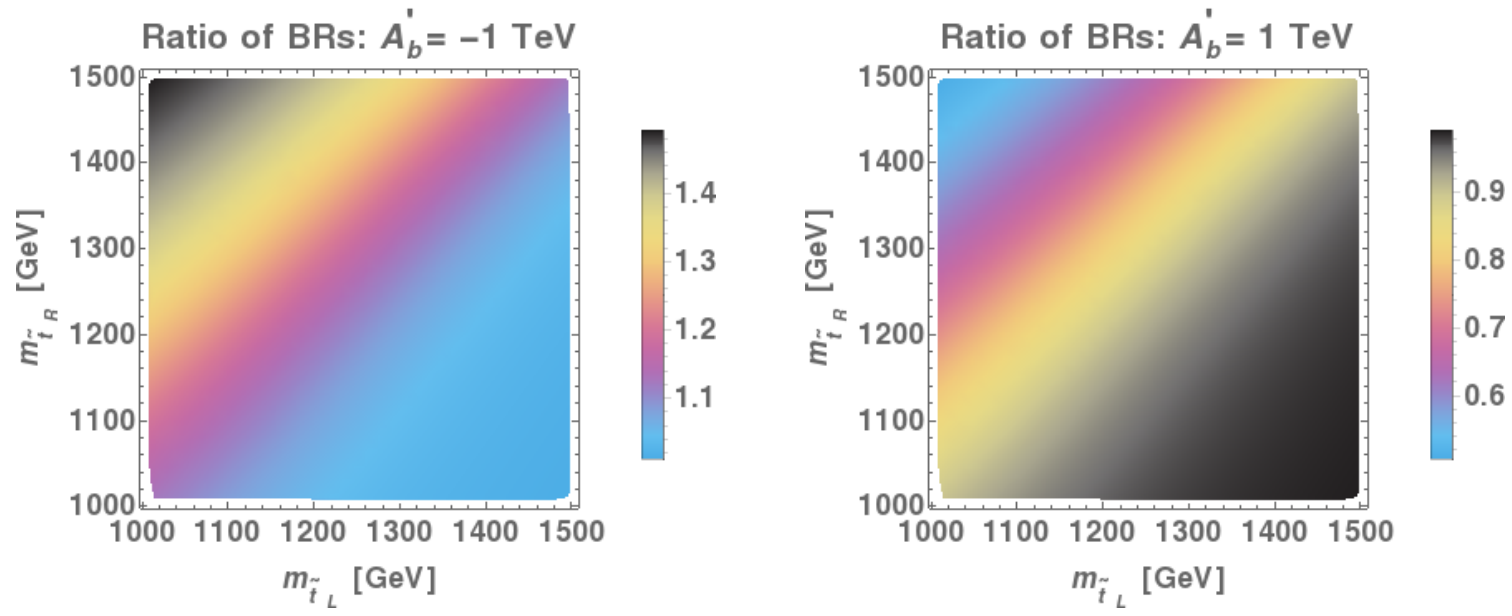

Figure 17. Variations of the ratio of branching fractions for the decay $\tilde{t}_{1} \rightarrow b \chi_{1}^{+}$in the NHSSM and the MSSM in the $\tilde{t}_{L}-\tilde{t}_{R}$ plane for $A_{b}^{\prime}=-1 \mathrm{TeV}$ (left) and $A_{b}^{\prime}=1 \mathrm{TeV}$ (right) and for fixed values of $\tan \beta(=40)$ and $\mu(=200 \mathrm{GeV})$. Other fixed parameters are as in figure 16 .

soft $\mathrm{NH}$ terms on the masses and mixings of the stops cannot be large since this is going to be $\tan \beta$-suppressed. However, as indicated there, it remains to be seen if an altered $y_{b}$ could play any role in the decays of stops. Indeed, an expression analogous to equation (3.2) for the $\tilde{t}_{i}-b-\chi_{1}^{+}$vertex would dictate that a left-like stop would couple to a higgsino-like chargino and a bottom quark with the strength $y_{b}$. Hence its decay rate to $b \chi_{1}^{+}$could get modified when compared to its MSSM expectation depending upon the altered value of $y_{b}$ as functions of $A_{b}^{\prime}$ and $\tan \beta$.

In figure 17 we illustrate the variations of the relative branching rate of $\tilde{t}_{1}$ to $b \chi_{1}^{+}$in the NHSSM (with respect to the MSSM case) in the plane of $\tilde{t}_{L}$ and $\tilde{t}_{R}$ for two values of 
$A_{b}^{\prime}(-1 \mathrm{TeV}$ (left) and $1 \mathrm{TeV}$ (right)) and for $\tan \beta=40$ for which the changes in the values of $y_{b}$ are moderately large. It is clear from these plots that when $\tilde{t}_{1}$ is $\tilde{t}_{L}$-dominated (i.e., when $m_{\tilde{t}_{L}} \ll m_{\tilde{t}_{R}}$ ), the effect of altered $y_{b}$ is maximal. For $A_{b}^{\prime}<0$ (left plot) leading to $y_{b}$ which is larger than its MSSM expectation, the effect is manifested in an enhanced (the black patch) branching fraction in the top left region. The reverse is true for the right plot where the said region now suffers the most due to a diminished $y_{b}$ (the blue patch) as is expected for $A_{b}^{\prime}>0$.

\section{Conclusions}

Deciphering imprints of the NHSSM scenario at colliders would not be a simple proposition. Given the way the two important classes of nonholomorphic soft terms in the form of $\mu^{\prime}$ and $A_{f}^{\prime}$ appear in the NHSSM Lagrangian, perhaps the only plausible way to extract information about them is to undertake a thorough, precise study of the interactions of the sfermions with the electroweakinos.

In the present work, we mostly adopt a scenario in which the SUSY conserving parameter ' $\mu$ ' has a relatively small value $(\leq 350 \mathrm{GeV})$ which help keep the scenario 'natural'. Furthermore, we prefer the masses of the low-lying electroweakinos to be governed by ' $\mu$ ' and hence turning out to be higgsino-like. This then could exploit the dominant Yukawa couplings thus bringing the sfermions from the third generation into the folds, which could potentially be the lightest of the sfermions and hence could be within the reach of the LHC.

We find the sbottom sector to be especially sensitive to the NHSSM soft terms thanks to a very prominent dependence of the bottom Yukawa coupling $y_{b}$ on the trilinear soft $\mathrm{NH}$ parameter $A_{b}^{\prime}$ due to radiative effects, reinforced by possible large values of $\tan \beta$. This could have a moderate to large (and hence phenomenologically important) effect on the masses and the mixings of sbottoms. For example, while in the MSSM, the mass-split between the two sbottom states could only be around 1-2\% of the degenerate chiral soft masses in the most favorable scenario, one could achieve a $10-15 \%$ split in the NHSSM. In addition, the sbottoms, when produced in pairs at the LHC, might lead to a $2 b+\mathbb{E}_{T}$ final state via one-step decays of the sbottoms. Such a final state is being intensively looked for at the LHC experiments. This is in contrast to pair-produced light stops leading to top quarks or charginos in their decays both of which, in turn, undergo cascades giving rise to myriad possibilities in the final state involving intricate model-dependencies and in cases, having involved SM backgrounds.

In this work, we study in much detail the couplings of the sbottoms with the electroweakinos which exploit to the fullest the simultaneous dependence of $y_{b}$ on $A_{b}^{\prime}$ and $\tan \beta$ along with benefiting from specific chiral imprints of the sbottom states. For large, negative $A_{b}^{\prime}$ and large $\tan \beta$, we demonstrate that the NHSSM scenario could lead to a much healthier event rate in the $2 b+\mathbb{E}_{T}$ final state when compared to the MSSM for similar masses for the sbottom(s) and the LSP neutralino. The reverse is also possible for large positive $A_{b}^{\prime}$ thus ending up with a lower event count than what is expected in the MSSM. We find that under favorable circumstances, with sbottom masses not exceeding $\sim 1.5 \mathrm{TeV}$, $300 \mathrm{fb}^{-1}$ of LHC data could be sensitive to such excesses. On the other hand, we note that 
a possible depletion in the event count could only be convincingly established by the high luminosity run of the LHC. A suitably designed multi-channel study could prove to be more efficient in search for a powerful discriminator in the present exercise.

Last but not the least, we have demonstrated how the dependence of $y_{b}$ on $A_{b}^{\prime}$ could have an important bearing on the phenomenology of the stops at the LHC due to its altered branching fractions triggered by modified $y_{b}$.

\section{Acknowledgments}

AD acknowledges the hospitality of the School of Physical Sciences, Indian Association for the Cultivation of Science, Kolkata on several occasions during the course of the present work. SM acknowledges the hospitality of Harish-Chandra Research Institute, Allahabad during a collaborative visit during the same period. AKS acknowledges the support received from Department of Science and Technology, Government of India with fellowship (SERB NPDF) reference number PDF/2017/002935.

Open Access. This article is distributed under the terms of the Creative Commons Attribution License (CC-BY 4.0), which permits any use, distribution and reproduction in any medium, provided the original author(s) and source are credited.

\section{References}

[1] ATLAS collaboration, Observation of a new particle in the search for the Standard Model Higgs boson with the ATLAS detector at the LHC, Phys. Lett. B 716 (2012) 1 [arXiv:1207.7214] [INSPIRE].

[2] CMS collaboration, Observation of a new boson at a mass of $125 \mathrm{GeV}$ with the CMS experiment at the LHC, Phys. Lett. B $\mathbf{7 1 6}$ (2012) 30 [arXiv:1207.7235] [INSPIRE].

[3] H.P. Nilles, Supersymmetry, Supergravity and Particle Physics, Phys. Rept. 110 (1984) 1 [INSPIRE].

[4] J.D. Lykken, Introduction to supersymmetry, in Fields, strings and duality. Proceedings, Summer School, Theoretical Advanced Study Institute in Elementary Particle Physics, TASI'96, Boulder, U.S.A., June 2-28, 1996, pp. 85-153, hep-th/9612114 [INSPIRE].

[5] J. Wess and J. Bagger, Supersymmetry and supergravity, Princeton University Press, Princeton U.S.A., (1992).

[6] M. Drees, R. Godbole and P. Roy, Theory and phenomenology of sparticles: An account of four-dimensional N=1 supersymmetry in high energy physics, World Scientific, Hackensack, U.S.A., (2004).

[7] H. Baer and X. Tata, Weak scale supersymmetry: From superfields to scattering events, Cambridge University Press, U.K., (2006).

[8] H.E. Haber and G.L. Kane, The Search for Supersymmetry: Probing Physics Beyond the Standard Model, Phys. Rept. 117 (1985) 75 [INSPIRE].

[9] S.P. Martin, A supersymmetry primer, Adv. Ser. Direct. High Energy Phys. 21 (2010) 1 [Adv. Ser. Direct. High Energy Phys. 18 (1998) 1] [hep-ph/9709356] [INSPIRE]. 
[10] D.J.H. Chung, L.L. Everett, G.L. Kane, S.F. King, J.D. Lykken and L.-T. Wang, The soft supersymmetry breaking Lagrangian: Theory and applications, Phys. Rept. 407 (2005) 1 [hep-ph/0312378] [INSPIRE].

[11] J.R. Ellis, K. Enqvist, D.V. Nanopoulos and F. Zwirner, Observables in Low-Energy Superstring Models, Mod. Phys. Lett. A 1 (1986) 57 [inSPIRE].

[12] R. Barbieri and G.F. Giudice, Upper Bounds on Supersymmetric Particle Masses, Nucl. Phys. B 306 (1988) 63 [inSPIRE].

[13] R. Barbieri and A. Strumia, The 'LEP paradox', in 4th Rencontres du Vietnam: Physics at Extreme Energies (Particle Physics and Astrophysics) Hanoi, Vietnam, July 19-25, 2000, hep-ph/0007265 [INSPIRE].

[14] G.F. Giudice, Naturalness after LHC8, PoS(EPS-HEP2013) 163 (2013) [arXiv:1307.7879] [INSPIRE].

[15] https://twiki.cern.ch/twiki/bin/view/AtlasPublic/SupersymmetryPublicResults.

[16] https://twiki.cern.ch/twiki/bin/view/CMSPublic/PhysicsResultsSUS.

[17] B. de Carlos and J.A. Casas, One loop analysis of the electroweak breaking in supersymmetric models and the fine tuning problem, Phys. Lett. B 309 (1993) 320 [hep-ph/9303291] [INSPIRE].

[18] R. Kitano and Y. Nomura, A solution to the supersymmetric fine-tuning problem within the MSSM, Phys. Lett. B 631 (2005) 58 [hep-ph/0509039] [INSPIRE].

[19] M.E. Cabrera, J.A. Casas and R. Ruiz de Austri, Bayesian approach and Naturalness in MSSM analyses for the LHC, JHEP 03 (2009) 075 [arXiv: 0812.0536] [INSPIRE].

[20] D.M. Ghilencea and G.G. Ross, The fine-tuning cost of the likelihood in SUSY models, Nucl. Phys. B 868 (2013) 65 [arXiv: 1208.0837] [INSPIRE].

[21] D.M. Ghilencea, Fixing the EW scale in supersymmetric models after the Higgs discovery, Nucl. Phys. B 876 (2013) 16 [arXiv:1302.5262] [INSPIRE].

[22] D.M. Ghilencea, A new approach to Naturalness in SUSY models, PoS(Corfu2012) 034 (2013) [arXiv: 1304.1193] [INSPIRE].

[23] H. Baer, V. Barger, P. Huang, A. Mustafayev and X. Tata, Radiative natural SUSY with a 125 GeV Higgs boson, Phys. Rev. Lett. 109 (2012) 161802 [arXiv:1207.3343] [INSPIRE].

[24] H. Baer, V. Barger and D. Mickelson, How conventional measures overestimate electroweak fine-tuning in supersymmetric theory, Phys. Rev. D 88 (2013) 095013 [arXiv:1309.2984] [INSPIRE].

[25] M.W. Cahill-Rowley, J.L. Hewett, A. Ismail and T.G. Rizzo, The Higgs Sector and Fine-Tuning in the pMSSM, Phys. Rev. D 86 (2012) 075015 [arXiv:1206.5800] [INSPIRE].

[26] M.W. Cahill-Rowley, J.L. Hewett, A. Ismail and T.G. Rizzo, More energy, more searches, but the phenomenological MSSM lives on, Phys. Rev. D 88 (2013) 035002 [arXiv: 1211.1981] [INSPIRE].

[27] M. Cahill-Rowley et al., Complementarity of dark matter searches in the phenomenological MSSM, Phys. Rev. D 91 (2015) 055011 [arXiv:1405.6716] [INSPIRE].

[28] M. Perelstein and C. Spethmann, A collider signature of the supersymmetric golden region, JHEP 04 (2007) 070 [hep-ph/0702038] [INSPIRE]. 
[29] C. Boehm, P.S.B. Dev, A. Mazumdar and E. Pukartas, Naturalness of Light Neutralino Dark Matter in pMSSM after LHC, XENON100 and Planck Data, JHEP 06 (2013) 113 [arXiv: 1303.5386] [INSPIRE].

[30] L. Girardello and M.T. Grisaru, Soft Breaking of Supersymmetry, Nucl. Phys. B 194 (1982) 65 [INSPIRE].

[31] K.L. Chan, U. Chattopadhyay and P. Nath, Naturalness, weak scale supersymmetry and the prospect for the observation of supersymmetry at the Tevatron and at the CERN LHC, Phys. Rev. D 58 (1998) 096004 [hep-ph/9710473] [INSPIRE].

[32] S.P. Martin, Dimensionless supersymmetry breaking couplings, flat directions and the origin of intermediate mass scales, Phys. Rev. D 61 (2000) 035004 [hep-ph/9907550] [INSPIRE].

[33] U. Chattopadhyay, D. Das and S. Mukherjee, Exploring Non-Holomorphic Soft Terms in the Framework of Gauge Mediated Supersymmetry Breaking, JHEP 01 (2018) 158 [arXiv: 1710.10120] [INSPIRE].

[34] H.E. Haber and J.D. Mason, Hard supersymmetry-breaking 'wrong-Higgs' couplings of the MSSM, Phys. Rev. D 77 (2008) 115011 [arXiv:0711.2890] [InSPIRE].

[35] J. Bagger and E. Poppitz, Destabilizing divergences in supergravity coupled supersymmetric theories, Phys. Rev. Lett. 71 (1993) 2380 [hep-ph/9307317] [INSPIRE].

[36] U. Ellwanger, Nonrenormalizable Interactions From Supergravity, Quantum Corrections And Effective Low-Energy Theories, Phys. Lett. B 133 (1983) 187 [INSPIRE].

[37] I. Jack and D.R.T. Jones, Nonstandard soft supersymmetry breaking, Phys. Lett. B 457 (1999) 101 [hep-ph/9903365] [INSPIRE].

[38] I. Jack and D.R.T. Jones, Quasiinfrared fixed points and renormalization group invariant trajectories for nonholomorphic soft supersymmetry breaking, Phys. Rev. D 61 (2000) 095002 [hep-ph/9909570] [INSPIRE].

[39] J.P.J. Hetherington, The spectrum of the MSSM with nonstandard supersymmetry breaking, JHEP 10 (2001) 024 [hep-ph/0108206] [INSPIRE].

[40] A. Sabanci, A. Hayreter and L. Solmaz, Higgs Boson Masses Of The MSSM With General Soft Breaking, Phys. Lett. B 661 (2008) 154 [arXiv:0801.2029] [INSPIRE].

[41] E. Cincioglu, A. Hayreter, A. Sabanci and L. Solmaz, Loop corrections to the neutral Higgs bosons of the non-holomorphic supersymmetric standard model with explicit CP-violation, arXiv:0905.0726 [INSPIRE].

[42] C.S. Ün, Ş. H. Tanyıldızı, S. Kerman and L. Solmaz, Generalized Soft Breaking Leverage for the MSSM, Phys. Rev. D 91 (2015) 105033 [arXiv:1412.1440] [InSPIRE].

[43] G.G. Ross, K. Schmidt-Hoberg and F. Staub, On the MSSM Higgsino mass and fine tuning, Phys. Lett. B 759 (2016) 110 [arXiv: 1603.09347] [INSPIRE].

[44] G.G. Ross, K. Schmidt-Hoberg and F. Staub, Revisiting fine-tuning in the MSSM, JHEP 03 (2017) 021 [arXiv: 1701.03480] [INSPIRE].

[45] MSSM Working Group collaboration, A. Djouadi et al., The minimal supersymmetric standard model: Group summary report, in GDR (Groupement De Recherche) Supersymetrie Montpellier, France, April 15-17, 1998, 1998, hep-ph/9901246 [INSPIRE].

[46] U. Chattopadhyay and A. Dey, Probing Non-holomorphic MSSM via precision constraints, dark matter and LHC data, JHEP 10 (2016) 027 [arXiv:1604.06367] [INSPIRE]. 
[47] J. Beuria and A. Dey, Exploring Charge and Color Breaking vacuum in Non-Holomorphic MSSM, JHEP 10 (2017) 154 [arXiv:1708.08361] [INSPIRE].

[48] A.H. Chamseddine, R.L. Arnowitt and P. Nath, Locally Supersymmetric Grand Unification, Phys. Rev. Lett. 49 (1982) 970 [INSPIRE].

[49] R. Barbieri, S. Ferrara and C.A. Savoy, Gauge Models with Spontaneously Broken Local Supersymmetry, Phys. Lett. B 119 (1982) 343 [INSPIRE].

[50] L.J. Hall, J.D. Lykken and S. Weinberg, Supergravity as the Messenger of Supersymmetry Breaking, Phys. Rev. D 27 (1983) 2359 [InSPIRE].

[51] P. Nath, R.L. Arnowitt and A.H. Chamseddine, Gauge Hierarchy in Supergravity Guts, Nucl. Phys. B 227 (1983) 121 [inSPIRE].

[52] N. Ohta, Grand Unified Theories Based On Local Supersymmetry, Prog. Theor. Phys. 70 (1983) 542 [INSPIRE].

[53] G.F. Giudice and R. Rattazzi, Theories with gauge mediated supersymmetry breaking, Phys. Rept. 322 (1999) 419 [hep-ph/9801271] [INSPIRE].

[54] I. Jack, D.R.T. Jones and A.F. Kord, $R$ parity violation and general soft supersymmetry breaking, Phys. Lett. B 588 (2004) 127 [hep-ph/0402045] [inSPIRE].

[55] T. Hambye, E. Ma and U. Sarkar, Leptogenesis from neutralino decay with nonholomorphic R-parity violation, Nucl. Phys. B 590 (2000) 429 [hep-ph/0006173] [INSPIRE].

[56] J. Chakrabortty and S. Roy, Resonant Leptogenesis with nonholomorphic R-Parity violation and LHC Phenomenology, Phys. Rev. D 85 (2012) 035014 [arXiv:1104.1387] [INSPIRE].

[57] J.L. Feng, K.T. Matchev and T. Moroi, Focus points and naturalness in supersymmetry, Phys. Rev. D 61 (2000) 075005 [hep-ph/9909334] [InSPIRE].

[58] S. Akula, M. Liu, P. Nath and G. Peim, Naturalness, Supersymmetry and Implications for LHC and Dark Matter, Phys. Lett. B 709 (2012) 192 [arXiv:1111.4589] [INSPIRE].

[59] H. Baer, V. Barger, P. Huang, D. Mickelson, A. Mustafayev and X. Tata, Radiative natural supersymmetry: Reconciling electroweak fine-tuning and the Higgs boson mass, Phys. Rev. D 87 (2013) 115028 [arXiv:1212.2655] [INSPIRE].

[60] A. Delgado, M. Quirós and C. Wagner, General Focus Point in the MSSM, JHEP 04 (2014) 093 [arXiv: 1402.1735] [INSPIRE].

[61] A. Mustafayev and X. Tata, Supersymmetry, Naturalness, and Light Higgsinos, Indian J. Phys. 88 (2014) 991 [arXiv: 1404.1386] [INSPIRE].

[62] J.A. Casas, J.M. Moreno, S. Robles, K. Rolbiecki and B. Zaldívar, What is a Natural SUSY scenario?, JHEP 06 (2015) 070 [arXiv: 1407.6966] [INSPIRE].

[63] H. Baer, V. Barger and M. Savoy, Upper bounds on sparticle masses from naturalness or how to disprove weak scale supersymmetry, Phys. Rev. D 93 (2016) 035016 [arXiv:1509.02929] [INSPIRE].

[64] Planck collaboration, P.A.R. Ade et al., Planck 2015 results. XIII. Cosmological parameters, Astron. Astrophys. 594 (2016) A13 [arXiv:1502.01589] [INSPIRE].

[65] L.J. Hall, R. Rattazzi and U. Sarid, The top quark mass in supersymmetric $\mathrm{SO}(10)$ unification, Phys. Rev. D 50 (1994) 7048 [hep-ph/9306309] [INSPIRE]. 
[66] R. Hempfling, Yukawa coupling unification with supersymmetric threshold corrections, Phys. Rev. D 49 (1994) 6168 [inSPIRE].

[67] M. Carena, M. Olechowski, S. Pokorski and C.E.M. Wagner, Electroweak symmetry breaking and bottom-top Yukawa unification, Nucl. Phys. B 426 (1994) 269 [hep-ph/9402253] [INSPIRE].

[68] D.M. Pierce, J.A. Bagger, K.T. Matchev and R.-j. Zhang, Precision corrections in the minimal supersymmetric standard model, Nucl. Phys. B 491 (1997) 3 [hep-ph/9606211] [INSPIRE].

[69] H.E. Logan, Supersymmetric Radiative Corrections at Large Tan $\beta$, Nucl. Phys. Proc. Suppl. 101 (2001) 279 [hep-ph/0102029] [INSPIRE].

[70] S. Antusch and M. Spinrath, Quark and lepton masses at the GUT scale including SUSY threshold corrections, Phys. Rev. D 78 (2008) 075020 [arXiv:0804.0717] [INSPIRE].

[71] A. Crivellin, Effective Higgs Vertices in the generic MSSM, Phys. Rev. D 83 (2011) 056001 [arXiv: 1012.4840] [INSPIRE].

[72] A. Crivellin, L. Hofer and J. Rosiek, Complete resummation of chirally-enhanced loop-effects in the MSSM with non-minimal sources of flavor-violation, JHEP 07 (2011) 017 [arXiv: 1103.4272] [INSPIRE].

[73] A. Crivellin and C. Greub, Two-loop supersymmetric QCD corrections to Higgs-quark-quark couplings in the generic MSSM, Phys. Rev. D 87 (2013) 015013 [Erratum ibid. D 87 (2013) 079901] [arXiv: 1210.7453] [INSPIRE].

[74] S. Kraml, Stop and sbottom phenomenology in the MSSM, hep-ph/9903257 [INSPIRE].

[75] F. Staub, SARAH 4: A tool for (not only SUSY) model builders, Comput. Phys. Commun. 185 (2014) 1773 [arXiv: 1309.7223] [INSPIRE].

[76] F. Staub, Exploring new models in all detail with SARAH, Adv. High Energy Phys. 2015 (2015) 840780 [arXiv: 1503.04200] [INSPIRE].

[77] W. Porod and F. Staub, SPheno 3.1: Extensions including flavour, CP-phases and models beyond the MSSM, Comput. Phys. Commun. 183 (2012) 2458 [arXiv:1104.1573] [InSPIRE].

[78] P. Bechtle et al., HiggsBounds-4: Improved Tests of Extended Higgs Sectors against Exclusion Bounds from LEP, the Tevatron and the LHC, Eur. Phys. J. C 74 (2014) 2693 [arXiv: 1311.0055] [INSPIRE].

[79] P. Bechtle, S. Heinemeyer, O. Stål, T. Stefaniak and G. Weiglein, HiggsSignals: Confronting arbitrary Higgs sectors with measurements at the Tevatron and the LHC, Eur. Phys. J. C 74 (2014) 2711 [arXiv:1305.1933] [INSPIRE].

[80] ATLAS collaboration, Search for supersymmetry in events with b-tagged jets and missing transverse momentum in pp collisions at $\sqrt{s}=13 \mathrm{TeV}$ with the ATLAS detector, JHEP 11 (2017) 195 [arXiv: 1708.09266] [INSPIRE].

[81] https://launchpad.net/mg5amcnlo.

[82] J. Alwall et al., The automated computation of tree-level and next-to-leading order differential cross sections and their matching to parton shower simulations, JHEP 07 (2014) 079 [arXiv: 1405.0301] [INSPIRE].

[83] LEPSUSYWG, ALEPH, DELPHI, L3 and OPAL experiments, note LEPSUSYWG/01-03.1, http://lepsusy.web.cern.ch/lepsusy/Welcome.html. 